OPEN ACCESS

Edited by:

Qian Zhong,

Sun Yat-sen University Cancer Center (SYSUCC), China

Reviewed by:

Damya Laoui,

Vrije University Brussel,

Belgium

Chiara Porta

University of Eastern Piedmont,

Italy

*Correspondence:

Chuanyu Sun

zhugexianglong@163.com

Guowei Xia

xiaguowei@fudan.edu.cn

Fan Zhong

zonefan@163.com

${ }^{\dagger}$ These authors have contribution

equally to this work

Specialty section:

This article was submitted to Molecular and Cellular Oncology,

a section of the journal

Frontiers in Oncology

Received: 11 April 2021

Accepted: 24 May 2021

Published: 10 June 2021

Citation:

Tan Y, Wang M, Zhang Y, Ge S,

Zhong F, Xia G and Sun C (2021)

Tumor-Associated Macrophages: A

Potential Target for Cancer Therapy.

Front. Oncol. 11:693517.

doi: 10.3389/fonc.2021.693517

\section{Tumor-Associated Macrophages: A Potential Target for Cancer Therapy}

\author{
Yifan $\operatorname{Tan}^{1 \dagger}$, Min Wang ${ }^{2 \dagger}$, Yang Zhang ${ }^{3}$, Shengyang Ge ${ }^{1}$, Fan Zhong ${ }^{3 *}$, Guowei Xia ${ }^{1 *}$ \\ and Chuanyu Sun ${ }^{\text {* }}$
}

${ }^{1}$ Department of Urology, Huashan Hospital, Fudan University, Shanghai, China, ${ }^{2}$ Department of Urology, Renmin Hospital of Wuhan University, Wuhan, China, ${ }^{3}$ Department of Systems Biology for Medicine, Institutes of Biomedical Sciences, Shanghai Medical College, Fudan University, Shanghai, China

Macrophages, an important class of innate immune cells that maintain body homeostasis and ward off foreign pathogens, exhibit a high degree of plasticity and play a supportive role in different tissues and organs. Thus, dysfunction of macrophages may contribute to advancement of several diseases, including cancer. Macrophages within the tumor microenvironment are known as tumor-associated macrophages (TAMs), which typically promote cancer cell initiation and proliferation, accelerate angiogenesis, and tame anti-tumor immunity to promote tumor progression and metastasis. Massive infiltration of TAMs or enrichment of TAM-related markers usually indicates cancer progression and a poor prognosis, and consequently tumor immunotherapies targeting TAMs have gained significant attention. Here, we review the interaction between TAMs and cancer cells, discuss the origin, differentiation and phenotype of TAMs, and highlight the role of TAMs in pro-cancer functions such as tumor initiation and development, invasive metastasis, and immunosuppression. Finally, we review therapies targeting TAMs, which are very promising therapeutic strategies for malignant tumors.

Keywords: tumor-associated macrophages, tumor microenvironment, cancer immunotherapy, macrophages, cancer

\section{INTRODUCTION}

Cancer is an important public health problem and the second leading cause of death, with approximately $14.1 \times 10^{6}$ new cancer cases and $8.2 \times 10^{6}$ deaths worldwide each year (1). Lung, breast and colorectal cancers are the most commonly diagnosed neoplasms, while the top three leading causes of death are from malignancy of the lung, liver and stomach $(1,2)$. Although advances in treatment strategies have resulted in an increase in overall survival rates for many cancers, some patients may experience recurrence or even distant metastases, leading to advanced stages of the disease (3). Thus, a better understanding of tumor biology is critical for the development of novel therapeutic strategies for cancer patients. The pathogenesis of cancer is intricate, involving not only alterations in the genomes of tumor cells, but also in the microenvironment in which they reside (4). The tumor microenvironment (TME), containing immune cells (e.g., neutrophils, macrophages, $\mathrm{T}$ cells etc.), fibroblasts, endothelial cells, secreted molecules and extracellular matrix, plays an important role in promoting tumor progression and has therapeutic potential (5). Among these cells, macrophages are a prominent component of the 
tumor microenvironment, representing an evolutionarily ancient cell type involved in tissue homeostasis and immune response to pathogens (6). These cells exhibit a high degree of plasticity in response to various external signals and are involved in both innate and adaptive immune responses; Under certain conditions, including stimulation by cytokines, macrophages are polarized into different phenotypes, classically activated macrophages (M1) and alternatively activated macrophages (M2) (7). However, recent research suggests that such traditionally nomenclature is too simplistic, as macrophages can express overlapping M1 and M2 gene (8). A better approach would be to describe it as a dynamic phenotypic spectrum, with M1 and M2 macrophages being the two extremes of this spectrum (9). Moreover, macrophages within the tumor microenvironment (known as tumor-associated macrophages) tend to be a different profile and their functional phenotypes are mainly determined by the surrounding context; therefore, TAMs are heterogeneous and exert a mixture of phenotypic characteristics, with not only the two extremes of a spectrum, namely M1 like macrophages and M2 like macrophages, but also other unknown polarized macrophage sets (10).

Previous evidences have suggested that massive infiltration of TAM or enrichment of TAM-related genes usually indicates tumor progression and a poor prognosis (11-13). In contrast, infiltration of TAM has also been found to predict a good prognosis in certain cancers, such as colorectal and ovarian carcinomas $(14,15)$. Indeed, TAMs play a dual role in tumors; they may exert pro-cancer effects via various pathways, such as by promoting tumor cell development, inducing tumor angiogenesis, promoting tumor cell metastasis and invasion, mediating resistance to drug therapy, and depressing antitumor immune responses (16). On the other hand, TAMs can be activated or reprogramed to trigger anti-tumor activities by secreting immunocidal molecules (e.g. ROS) and inflammatory cytokines (e.g. IFN- $\gamma$ and TNF- $\alpha$ ), or directly phagocytosing neoplastic cells and recruiting of tumor-killing leukocytes or activating adaptive immune responses $(10,17)$. Thus, these findings suggest that targeting TAMs and molecules associated with them could be a strategy for cancer treatment (16). In particular, the reprogramming of TAMs has attracted much attention. Here, we review the relationship between TAMs and cancer, discuss the origin, differentiation and phenotype of TAMs, and focus on the pro-tumor function of TAMs. Finally, we discuss therapies targeting TAMs, which are very promising strategies for cancer treatment.

\section{MOLECULAR CHARACTERISTICS OF TAMs}

\section{Origin of TAMs}

The origin of macrophages remains inconclusive. However, with the application of modern lineage tracing technology, the understanding of their origin has changed dramatically, macrophages actually derive from at least three sources: including the bone marrow (BM), the fetal liver and the embryonic yolk sac $(16,18)$. There are two general types of macrophages based on their origin and resident location, namely bone-marrow-derived macrophages (BMDMs) and tissueresident macrophages (TRMs) (18). In general, hematopoietic stem cells (HSCs) in the bone marrow give rise to BMDMs, HSCs first develop into promonocytes and then enter the peripheral blood where they develop into mature monocytes, and finally exit capillaries and enter into the tissues to develop into tissuespecific macrophages (19). Nevertheless, the vast majority of TRMs originate from colony-stimulating factor 1 receptor (CSF1-R)-positive erythro-myeloid progenitors (EMPs) in the embryonic yolk sac or the fetal liver, which are different from BMDMs $(18,20)$. For a long time, it was believed that TRMs seeded in different tissue were gradually replenished and replaced by monocytes, which derived from HSCs in the adult BM. However, recent evidences indicate that TRMs are not replaced during embryonic development and that they proliferate locally and self-maintain independently throughout adulthood with little involvement of HSCs (21-23). In particular, microglia in the brain, a subset of TRMs, whose sole origin appears to be yolk sac-derived EMPs, seem never to be replaced by subsequently myeloid progenitors arising in fetal or adult life $(23,24)$. In tumor tissues, TAMs originate from either bone-marrow-derived macrophages or tissue-resident macrophages (Figure 1), but the proportion of TRM and BMDM in tumors varies considerably according to their tissue and organ specificity (25-27). Studies have reported that in most cases, TAMs largely originate from monocytes in the blood and are recruited into tumor tissue via chemokines (26). For example, in models of breast cancer, $\mathrm{CCR}^{+}$monocytes are the main source of TAMs, accounting for approximately $40 \%$ of all $\mathrm{CD} 45^{+}$cells within tumor tissues, compared to less than $10 \%$ derived from TRMs (26). In contrast, in pediatric solid tumors of fetal and postnatal developmental origin such as retinoblastoma, neuroblastoma, and osteosarcoma, TAMs are primarily derived from tissue-resident macrophages (25). Moreover, different sources of TAMs in the same tissue may exert different effects. For instance, in pancreatic ductal adenocarcinoma, TAMs are also mainly derived from embryonic-derived TRMs, which are more favorable for tumor cell proliferation and progression compared to monocytederived TAMs; in animal models, removal of tissue-resident macrophages can inhibit tumor progression (27). Another study on lung cancer indicates that monocyte-derived TAMs are correlate with promoting tumor spreading, while TRMs are associate with tumor growth in vivo (28). Therefore, these findings imply that both BMDMs and TRMs are present at different levels in different tumor models, and that the origin of TAMs has a potential influence on their functional changes, which suggests that targeting TAMs as a therapeutic strategy should be analyzed according to their origin and tissue organ and tumor specificity.

\section{Regulation of TAM Differentiation}

Macrophages are regulated by a series of transcription factors during differentiation, among which PU.1 (also known as SPI1) plays a dominant role (29). However, macrophages from 


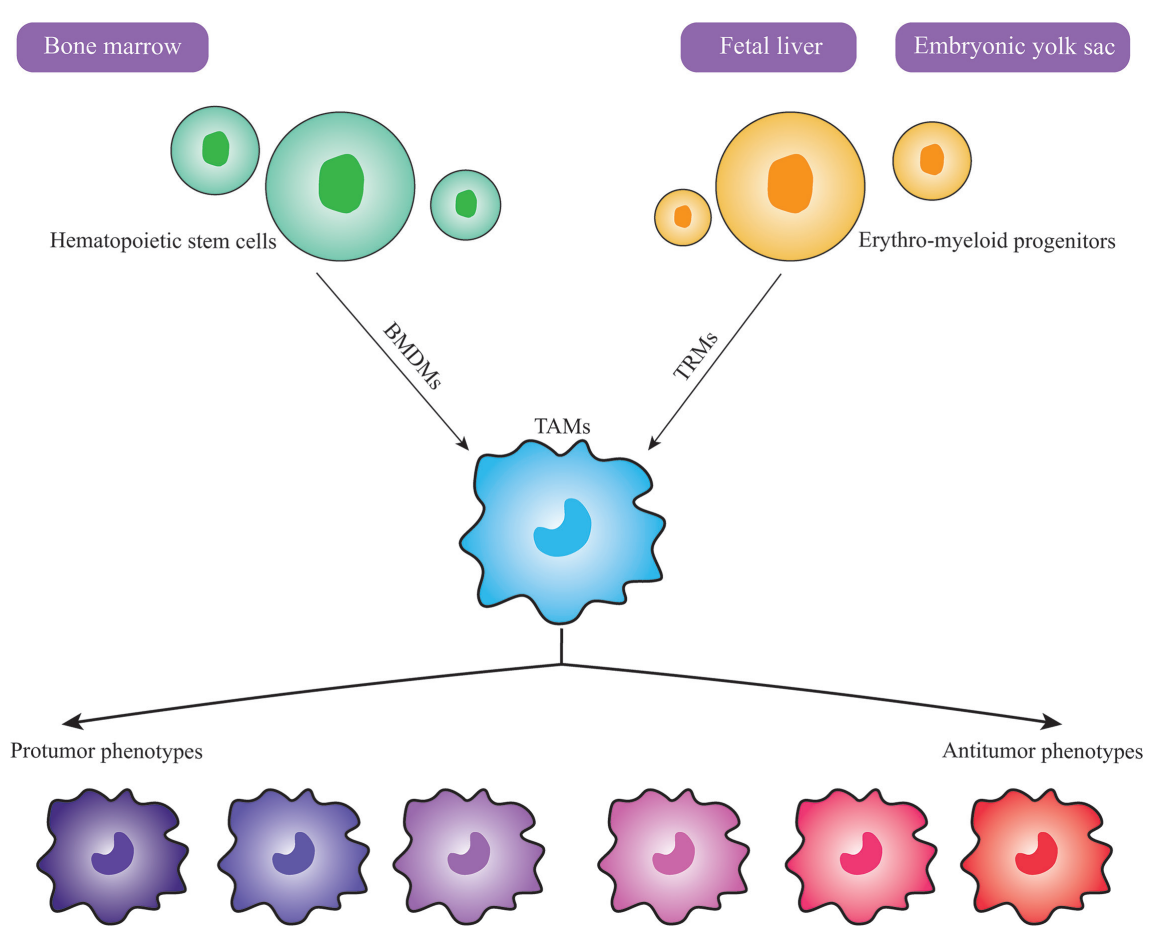

FIGURE 1 | Origin and Heterogeneity of TAMs. Macrophages in tumors actually have at least three sources, including bone marrow, fetal liver and embryonic yolk sac. Bone-marrow-derived macrophages (BMDMs) develop from hematopoietic stem cells in the bone marrow, while tissue-resident macrophages (TRMs) develop from erythro-myeloid progenitors in the fetal liver or embryonic yolk sac. TAMs can differentiate into various phenotypes upon stimulation by different signals in the tumor microenvironment, and the protumor and antitumor phenotypes are the two extremes of the spectrum.

different tissues (e.g., lung, liver, or brain) exhibit a highly diverse transcriptional landscape (30). For instance, after in-vivo purified peritoneal macrophages were transplanted into the lungs of mice, they acquired a gene expression profile similar to that of alveolar macrophages (30). It was suggested that macrophage differentiation in tissues occurs locally and their regulation is driven to a large extent by the environment in which they reside. A similar situation may apply to TAMs, where the majority of TAMs in human tumors originate mainly from myeloid monocytes that are recruited to the tumor tissue and undergo a series of functional and phenotypic transformations to acquire TAM properties (31). There are also changes in expression of surface molecules, especially of mannose receptor (CD206), scavenger receptor (CD163) and arginase-1, which are expressed at higher levels (32). The transcriptional landscape of TAMs is not dependent on PU.1, but is regulated by signaling molecules from multiple sources, including signals form tumor and normal tissues; all off these signals must interact with developmental cues that control macrophage differentiation and ultimately mediate the properties and heterogeneity of TAMs (25). For example, in a mammary tumor model, unlike that of normal tissue macrophages, terminal differentiation of TAMs is dependent on activation of the Notch signaling pathway mediated by the transcriptional regulator RBPJ; furthermore, intervention in the Notch signaling pathway can have an antitumor effect by altering differentiation and maturation of TAMs
(26). In contrast, knocking down RBPJ and thus interfering with the Notch signaling pathway not only failed to inhibit tumor growth, but also accelerated tumor proliferation in a hepatocellular carcinoma (33). In addition, the anti-cancer effect of type I interferon (IFN)-based therapies has been reported to target $\mathrm{Ly}_{6} \mathrm{C}^{+}$monocytes and hinder their differentiation into TAMs (34). However, TAMs are well known for their plasticity, which allows them to acquire various phenotypes in different microenvironments. Thus, the applicability of TAM-targeted therapeutic strategies to other types of malignancies remains to be determined.

\section{Phenotypes and Function of TAMs}

As an important class of innate immune cells, macrophages are recognized to be highly plastic and heterogeneous, and their phenotypes are regulated by the microenvironment in which they are located. For example, upon stimulation by proinflammatory factors such as lipopolysaccharide (LPS), IFN- $\gamma$, and tumor necrosis factor (TNF)- $\alpha$, macrophages activate into M1-type macrophages (classical activation); while exposed to anti-inflammatory stimuli, including interleukin (IL)4 and IL-13, they differentiated into M2-type macrophages (alternative activation) (7). When the body is invaded by pathogens, macrophages rapidly polarize into M1-type macrophages that secrete a variety of inflammatory factors (e.g., IL-6, IL-12, IL-1 $\beta$ and TNF- $\alpha$ ) to promote an 
inflammatory response, engulf and destroy pathogens, process and present antigens, and initiate an adaptive immune response $(7,35)$. However, if the M1 phase becomes extended, it can cause tissue damage. Thus, macrophages polarize into the M2 type, releasing large amounts of anti-inflammatory cytokines (IL-10 and TGF- $\beta$ ) that attenuate an excessive inflammatory response and contribute to tissue repair, angiogenesis and maintenance of body homeostasis (36). It should be noted that M1 and M2 macrophages are merely the two extreme descriptions of the polarization state of macrophages and there are also other polarized macrophage populations (9). For instance, M2 macrophages can be induced to transform into M2a, M2b, M2c, and M2d subtypes under different conditions (37). M2a and M2c macrophages are functionally similar in terms of antiinflammation, immunosuppression, and tissue repair, while M2b is more complex and associated with immune regulation; as for M2d macrophages, these cells are induced by co-stimulation by A2 adenosine receptor (A2R) and Toll-like receptor (TLR) agonists and are characterized by production of high levels of IL-10 and vascular endothelial growth factor (VEGF) and low levels of IL-12 and TNF- $\alpha$ (37-39). Some previous reviews had suggested that TAMs were equivalent to M2d macrophages (38). However, such classification of TAMs is difficult as this cell population is not a typical subgroup of macrophages and cannot be observed in the steady state, but is associated with specific pathological conditions, such as tumors and inflammation (40). Moreover, the phenotypic and functional heterogeneity of TAMs makes them highly variable between different tumors or even among different regions of the same tumor (41). Thus, the view that TAMs are equal to M2d macrophages is inaccurate.

Previous reports have indicated that TAMs can also be classified into M1-like macrophages (antitumor phenotype) and M2-like macrophages (protumor phenotype) (42, 43). Upon stimulation by proinflammatory factors (LPS, TNF- $\alpha$ ), TAMs activate into M1-like macrophages and kill tumor cells by producing reactive oxygen species and inflammatory cytokines (e.g. IL-6 and TNF- $\alpha$ ) (10). While in most types of tumor tissues, under the influence of signals originating from cancer cells or normal cells present in the TME, TAMs generally exhibit a higher degree of similarity to M2 macrophages and produce antiinflammatory cytokines and chemokines (e.g. TGF- $\beta$, IL-10, CCL18, CCL22) that have little cytotoxic effect on tumor cells, but possess pro-tumor properties $(16,18)$. This is not only due to the overexpression of M2-type polarizing signals (such as IL-10, IL-4, IL-13, and TGF- $\beta$ ) in the TME, but is also related to the fact that pro-inflammatory cytokines can also confer pro-tumor properties to TAMs. For example, IFN- $\gamma$ can directly enhance the expression of immunosuppressive enzymes such as indoleamine 2,3 dioxygenase (IDO) and nitric oxide synthase (NOS2) in TAMs, myeloid-derived suppressor cells (MDSCs), and dendritic cells $(44,45)$. Nevertheless, the M1 and M2 classification of TAMs mainly corresponds to in vitro conditions without environment influence, and TAMs in vivo are not so neatly divided into M1 and M2, especially within tumors (18). Thus, it is too simplistic to classify the phenotype and function of TAMs only from the perspective of M1 and M2 macrophages or based on a selection of markers. In addition, the gene signatures of TAMs in brain tumors are distinct from that of M1 and M2 in vitro polarized macrophages (46). Recent new technologies, such as single-cell RNA sequencing, multiplexed cytometry and mass cytometry by time-of-flight, also reveal that heterogeneity of TAMs may be more complex and diverse (47). Indeed, TAMs have various functional phenotypes and display remarkable plasticity and can play two opposing roles in cancer, including inhibiting and promoting tumor progression (Figure 1) (48). Additionally, TAMs have the ability to transform dynamically between antitumor and protumor phenotypes in respond to the flexible environment signals in the TME; this dramatically plasticity also leads to various subpopulation of TAMs (49). What's more, several studies have reported that distribution of TAMs in the TME, such as inter- and intra-tumoral of TAMs, may have different phenotypes and functions in the same tumor (50). Therefore, TAMs may be more appropriately defined according to their ontogeny, activation status, function, intratumoral and intertumoral localization (49). Although TAMs are intricately heterogeneous and their role still needs further investigation, TAMs have profound effects in tumors and can be considered in cancer immunotherapy.

\section{PRO-TUMOR PROPERTIES OF TAMS}

Over recent decades, a plethora of studies have shown that TAMs exert pro-tumor effects and are closely associated with tumor progression. In fact, it has been demonstrated that TAMs exert their pro-cancer effects via two pathways: 1) direct pro-tumor properties, such as participating in the initiation of tumorigenesis, promoting tumor progression, metastasis to distant sites, and inducing therapeutic resistance in tumor cells, and 2) indirect pro-tumor properties, such as inhibiting anti-tumor immune responses to avoid damage to tumor cells and indirectly supporting tumor progression. A schematic depiction of the effects of TAMs in tumors is shown in Figure 2.

\section{TAMs in Tumor Initiation}

During the $19^{\text {th }}$ century, it became apparent that a pathophysiological association between tumors and inflammation, especially long-term chronic inflammation, may contribute to initiation of cancers such as liver cancer, caused by the hepatitis B virus, and colon cancer, caused by inflammatory bowel disease $(51,52)$. Moreover, patients with chronic obstructive pulmonary disease (COPD), an inflammatory disease of the lungs, have an increased risk of lung carcinoma; bronchial inflammation induced by Haemophilus influenzae was also shown to increase the incidence of tumors in animal models of lung cancer $(53,54)$. In the microenvironment of chronic inflammation, there are a large number of inflammatory cells secreting various cytokines and growth factors, increasing the production of reactive substances, promoting oxidative DNA damage and reducing DNA repair, the net effect of which is a loss of normal cell damage control and repair. Thus, in a sense, 
tumors are like wounds that cannot be healed (55). Macrophages, as one of the most common immune cells in the tumor microenvironment, connect cancer and inflammation. During early carcinogenesis, TAMs are mainly characterized as proinflammatory macrophages, which induce the expression of inflammatory cytokines such as IL- 6 and TNF- $\alpha$; moreover, macrophages produce reactive oxygen and nitrogen species (ROS), and nitric oxide (NO) can react with peroxidase to produce nitrosoperoxycarbonate, further promoting inflammation and creating a mutagenic environment that causes mutations in normal epithelial cells (43). Furthermore, promoting inflammation by, e.g., silencing STAT3 in macrophages or inhibiting the expression of the antiinflammatory factor IL-10, can further induce tumorigenesis $(56,57)$. By contrast, in a model of intestinal cancer, removal of ROS produced in macrophages suppresses carcinogenesis (58). After tumor formation, TAMs generally exhibit antiinflammatory-associated markers such as arginase-1, CD206 and low levels of MHC-class II, and polarize into protumor macrophages that migrate into the tumor microenvironment, secreting growth factors that both suppress anti-tumor immune responses and support tumor cell proliferation (59). In mammary tumors, for example, TAMs secrete epidermal growth factor receptor (EGFR) family ligands, including heparin-binding EGF-like growth factor (HB-EGF), and activate STAT3-related signaling pathways to fuel tumor cell proliferation (60). Although not observed in all types of cancer, the pro-proliferative function of TAMs has been demonstrated in a variety of tumor studies, including pancreatic cancer, liver cancer, breast cancer, etc. $(27,33,60,61)$. For example, in hepatocellular carcinoma, blockade of the RBPJ-regulated Notch signaling pathway reduced infiltration of monocytederived TAMs into tumor tissues, but activated the WNT/ $\beta$ catenin signaling pathway, leading to massive infiltration of Kupffer cell-derived TAMs into hepatocellular carcinoma tissues, which also upregulated anti-inflammatory cytokine IL10 levels, downregulated IL-12 levels, and further facilitated tumor proliferation (33). In addition, it was shown that extracellularly regulated protein kinase 5 (ERK5) promoted macrophage polarization toward the protumor type, whereas silencing ERK5 expression in macrophages impaired STAT3 phosphorylation, induced TAM polarization toward the proinflammatory type, and exerted anti-tumor effects (61).

\section{TAMs in Tumor Progression}

In addition to participating in tumor initiation and promotion, TAMs also play an important role in the progression from benign to malignant cancer. For example, in breast tumors, CSF-1 may accelerate tumor progression and the transition to malignancy by recruiting TAM infiltration (62). As with normal cells, tumor cells also require a vascular network to provide nutrients and oxygen and to remove metabolic waste for maintaining activity and metabolism (63). One of the key features of the progression of benign tumors to malignancy is overactive angiogenesis of the vascular network, a process that usually requires the involvement of innate immune cells; additionally, macrophages are important pro-angiogenic cells in the TME (64). Thus, TAMs support tumor angiogenesis mainly by the production of pro-angiogenic factors. Furthermore, cancer cells can also secrete large amounts of

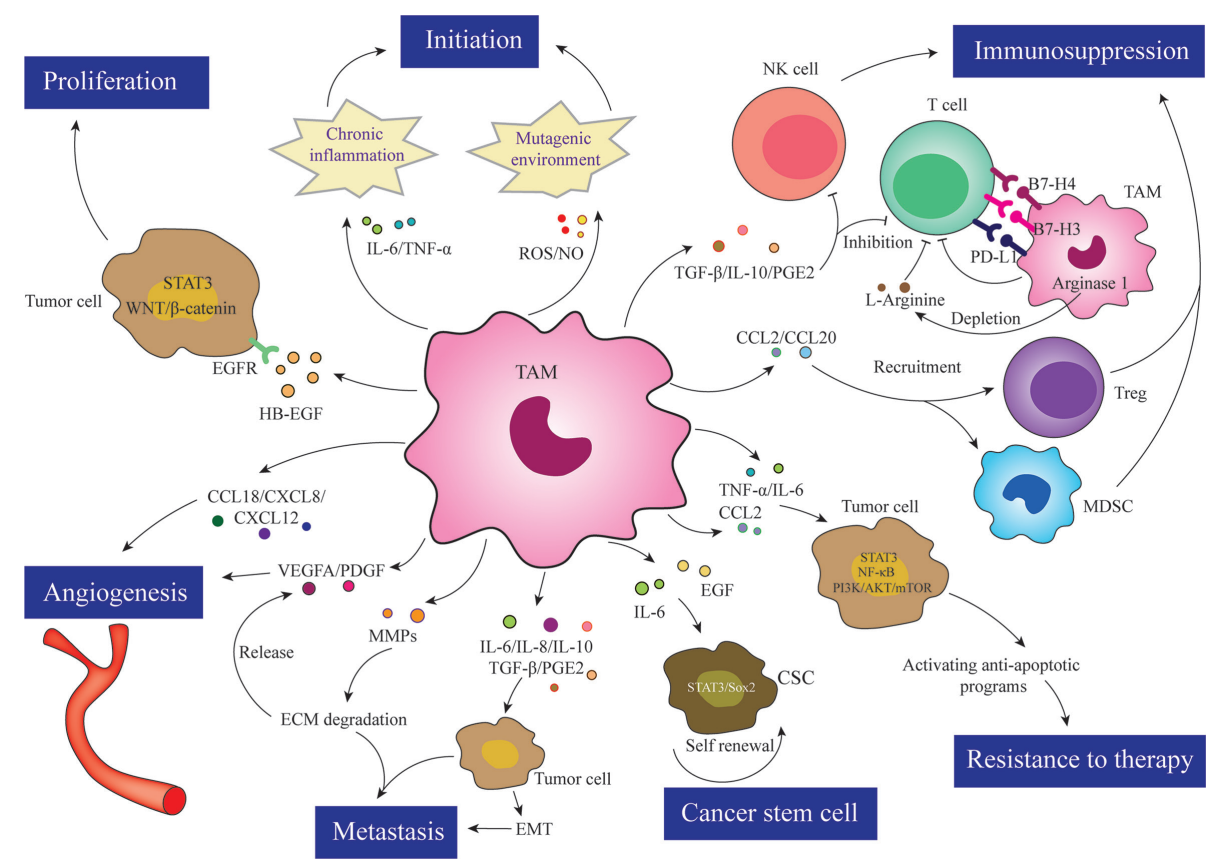

FIGURE 2 | TAMs are involved in almost all aspects of tumor cell biology through various mechanisms, such as tumor initiation, proliferation, angiogenesis, metastasis, immunosuppression, resistance to therapy, and cancer stem cell maintenance. 
angiopoietin-2, which promotes the recruitment of monocytes expressing the angiopoietin 1 receptor (TIE2) to the tumor site, leading to massive infiltration of TAMs into the cancerous tissue (65). In addition to secreting pro-angiogenic growth factors [such as vascular endothelial growth factor A (VEGFA), platelet-derived growth factor (PDGF) and angiogenic chemokines (CCL18, CXCL8, CXCL12)], TAMs are also a major source of matrix metalloproteinase 9 (MMP-9), which facilitates extracellular matrix degradation and further contributes to the release of VEGFA $(65,66)$. Ultimately, the tumor microenvironment contains high levels of VEGF. In turn, VEGF recruits vascular endothelial cells and macrophages into the tumor tissue, contributing to abnormal tumor vascular formation, including excessive branching, vascular leakage and dead vessels, that together affect tumor hemodynamics and chemotherapeutic drug delivery (67). VEGF antagonists significantly reverse the abnormal vascular phenotype in tumors, induce vascular normalization and increase the sensitivity of tumor cells to chemotherapy (68). Moreover, studies have also shown that TAM-secreted CCL18 enhances vascular endothelial cell migration as well as angiogenesis. Further analysis revealed that CCL8 interacts with its receptor PITPNM3 on the surface of human umbilical vein endothelial cells (HUVECs) and activates ERK and AKT/GSK-3 $\beta /$ Snail signaling pathways in HUVECs, thereby contributing to its pro-angiogenic effects, while blockading PITPNM3 on the surface of HUVECs with neutralizing antibodies inhibits endothelial cell migration and angiogenesis (66). Other factors such as TGF- $\beta$, TNF, WNT7B, and thymidine phosphorylase promote tumor progression by recruiting and activating endothelial or other cells (such as fibroblasts) that further support angiogenesis in the tumor microenvironment $(69,70)$.

\section{TAMs in Tumor Metastasis}

TAMs may trigger tumor initiation, accelerate tumor progression, and also contribute to cancer metastasis, leading to advanced stages of tumors. Tumor metastasis is characterized by cancer cells leaving the primary site and colonizing distant organs through blood or lymphatic vessels, which is one of the major causes of death in cancer patients (71). On the one hand, accumulating evidence has demonstrated that during metastasis, TAMs can induce epithelial-mesenchymal transitions (EMTs) in cancer cells, enhance tumor cell invasion, inhibit normal antigen presentation, reduce $\mathrm{T}$ cell recognition and destroy tumor cells by secreting various cytokines and inflammatory mediators such as IL-6, IL-8, IL-10, TGF- $\beta$ and prostaglandin E2; on the other hand, TAMs also provide matrix remodeling enzymes and cathepsins that disrupt the tumor stroma by upregulating metalloproteinases such as MMP7 and MMP9, facilitating tumor cell migration away from the primary site $(72,73)$. In a model of colorectal cancer, for example, research has shown that TAMs were induced by tumor cells to secrete IL- 6 that drove EMTs in tumor cells via the JAK2/STAT3/FoxQ1 axis, while in turn CCL2 produced by tumor cells recruited TAMs into the tumor microenvironment to form a vicious cycle that ultimately led to distant metastasis of colon cancer cells, and experiments in vivo revealed that inhibition of IL-6 significantly reduced tumor metastases (73). After leaving the primary site, tumor cells are attracted to blood vessels where they interact with perivascular $\mathrm{TIE}^{+}$macrophages, which increases vessel permeability and promotes tumor cell escape in part by secreting VEGFA (74). When invasive tumor cells enter the bloodstream, they need to survive in the blood or lymphatic circulation, avoid being recognized and eradicated by the immune system, reach distant organs and then colonize and grow in these normally hostile environments. Recent studies have shown that macrophages promote survival and growth of metastatic cancer cells by promoting the extravasation of invasive cancer cells from blood vessels, secreting signals that stimulate tumor cell growth and proliferation, and contributing to an immunosuppressive environment by suppression of cytotoxic T cell activity (75-77). Furthermore, it was found that CC-motif ligand 2 (CCL2) interacts with its receptor CCR2 to recruit inflammatory monocytes into the TME and become TAMs, which in turn can secrete another chemokine ligand, CCL3, to interact with metastatic cancer cells, improving TAM retention in metastatic foci and supporting tumor growth $(76,78)$. Moreover, in a lung metastasis model of breast cancer, metastatic cancer cells were observed to express vascular cell adhesion protein 1 (VCAM1) on their surface, which binds to $\alpha 4$-integrin, a molecular receptor on the surface of lung macrophages, which in turn activates the PI3K/Akt signaling pathway to maintain the survival of metastatic breast cancer cells in lung tissue (79).

\section{TAMs in Immunosuppression}

TAMs may exert anti-neoplastic effects when they differentiate into proinflammatory phenotype. However, in most types of cancer in which signals originate from cancer cells or normal cells present in the TME, TAMs exhibit a higher degree of similarity to anti-inflammatory macrophages and skew TAMs to a pro-tumor state (16). TAMs can induce immunosuppression and contribute to tumor immune escape via multiple mechanisms. Numerous studies have shown that TAMs secrete cytokines such as TGF- $\beta$, IL-10, and PGE2 (prostaglandin E2), and that these immunosuppressive cytokines directly suppress effector functions of $\mathrm{CD}^{+}$and $\mathrm{CD}^{+} \mathrm{T}$ cells, increase Treg (regulatory $\mathrm{T}$ ) cell expression and consequently contribute to an immunosuppressive microenvironment (80). In a model of breast cancer, for example, it was revealed that reducing PGE2 expression via deletion of cyclooxygenase-2 (COX-2) in TAMs could enhance $\mathrm{T}$ cell survival and immune surveillance and thereby suppress mammary tumor progression (81). Additionally, TAMs also cooperate with other immune cells to suppress anti-tumor immune responses. MDSCs (myeloidderived suppressor cells) and Tregs are two types of cells in the tumor microenvironment that mediate immunosuppression. TAMs produce chemokines such as CCL2 and CCL20, which recruit these cells into the tumor microenvironment $(82,83)$. For example, CCL2 expressed by TAMs in gliomas is essential for recruitment of CCR $4^{+}$Tregs and CCR2 ${ }^{+}$Ly- $6 \mathrm{C}^{+}$MDSCs (82). Furthermore, research has indicated that expression of $\mathrm{T}$ cell immune checkpoint ligands (e.g., PD-L1) in TAMs may be an important TAM-mediated immunosuppression mechanism 
(84). For example, TAMs isolated from a mouse model of bladder cancer expressed high levels of PD-L1, suppressing tumor-specific T-cell immunity and enhancing tumor growth (84). Moreover, TAMs also express B7 family proteins such as B7-H3 and B7-H4, and inhibition of these proteins with antibodies impaired tumor progression in a $\mathrm{CD}^{+} \mathrm{T}$ and $\mathrm{NK}$ cell-mediated manner $(85,86)$. L-arginine is an essential amino acid for the $\mathrm{CD} 3 \zeta$ chain in the $\mathrm{T}$ cell receptor complex that enhances the viability of activated $\mathrm{T}$ cells and the formation of memory $T$ cells $(87,88)$. Expression of Arginase 1 on TAMs accelerates the metabolism of L-arginine to urea and L-ornithine, thereby further dampening T-cell recognition of tumor antigens and the antitumor immune response (48). In addition, TAMs also express inhibitory molecules, including non-classical major histocompatibility complex (MHC) class I molecules (e.g. HLAE and HLA-G), which interact with inhibitory receptors (e.g. CD94 and leukocyte immunoglobulin-like receptor B subfamily member 1, LILRB1) on T and NK cells, to negatively regulate the activation of T and NK cells (89). Recent finding has implied that neoplastic cell can also express MHC class I molecules that interact with LILRB1 on TAMs and resist phagocytosis by TAMs, leading to loss of immune surveillance (90).

\section{TAMs in Other Pathways}

Cancer stem cells (CSC), a class of tumor cells with a stemness phenotype, are capable of self-renewal and have multidirectional differentiation potential, and play an important role in tumor development, invasion, metastasis, and resistance to treatment (91). Similar to the microenvironment of tumor cells, cancer stem cells have their own unique microenvironment, or "niche", consisting of fibroblasts and immune, perivascular and endothelial cells (92). TAMs, as key components of the CSC microenvironment, secrete cytokines and growth factors to support and promote the pro-cancer function of CSCs $(93,94)$. For example, research has shown that co-injection of CSCs and TAMs markedly increased tumor initiation activity and metastatic efficiency (93). Another study indicated that coculture of TAMs with hepatocellular carcinoma stem cells increased the expansion of CSCs and promoted their incorporation into spheroids in vitro and xenograft tumors in vivo, while tocilizumab blocked IL-6 signaling and reduced their oncogenic potential (94). Moreover, it has been demonstrated that TAMs are involved in induction and maintenance of the CSC niche. It was suggested that EGF (epidermal growth factor) secreted by TAMs interacts with EGF receptors on breast cancer cells to further activate the STAT3/Sox2 signaling pathway to induce the CSC stemness phenotype (95). In addition to regulating CSC self-renewal and maintenance, TAMs can also induce therapeutic resistance by secreting growth factors and chemokines and by activating anti-apoptotic programs in cancer cells (67). For example, the sensitivity of breast cancer cells to tamoxifen was significantly reduced after culture in media that had been used for TAMs, and addition of CCL2 to the culture medium further reduced the sensitivity of tumor cells to tamoxifen. Further analysis revealed that CCL2 secreted by TAMs activated the PI3K/AKT/mTOR pathway in tumor cells, thus inducing resistance to tamoxifen treatment in breast cancer cells (96). Another study showed that combined treatment with CSF-1 antibodies and chemotherapeutic agents enhanced the sensitivity of breast cancer tumor cells to chemotherapy by reducing TAM recruitment to the tumor microenvironment and inhibiting the expression of multidrug resistance gene 1, chemoresistance genes and matrix metalloproteinases (97).

\section{THERAPEUTIC STRATEGIES TARGETING TAMs}

Given the extensive involvement of TAMs in cancer cell biological processes, they are a potential target for cancer therapy. Currently, there are two main therapeutic strategies aimed at TAMs: (1) inhibition of TAM pro-cancer functions, including blockade of TAM recruitment or depletion of TAMs, and (2) "re-education" of TAMs to activate their anti-cancer function (Figure 3). Some drugs targeting TAMs in clinical trials are presented in Table $\mathbf{1}$.

\section{Inhibiting Pro-Tumor TAMs}

Blocking recruitment of macrophages into cancer tissue to reduce their pro-tumor effects is a promising therapeutic strategy. A plethora of cytokines and chemokines are involved in recruiting monocyte-derived macrophages into the tumor microenvironment, such as chemokine CCL2 $(26,76,78,96)$. Tumor cells and stromal cells in the tumor microenvironment can secrete CCL2, which interacts with its chemokine receptor CCR2 and exerts a critical role not only in recruiting bone marrowderived monocytes into tumors and differentiation of monocytes into TAMs, but also in recruiting other immunosuppressive cells such as MDSCs and regulatory T cells $(26,76,82)$. For example, in hepatocellular carcinoma, inhibition of CCL2 with specific monoclonal antibodies slows tumor progression and metastasis by blocking the recruitment of TAMs (78). Inactivation of neddylation was shown to significantly inhibit CCL2 secretion from lung cancer cells, reducing infiltration of monocytes into the tumor microenvironment and their development into TAMs, ultimately increasing survival (98). Thus, effective blockade of the CCL2/CCR2 axis is an effective way to restrict macrophage recruitment. At present, there are mainly two kinds of drugs being used in clinical trials: one is a CCL2-blocking antibody (carlumab, CNTO888), and the other is a small molecule inhibitor of CCR2 (PF-04136309) $(16,99)$. In a phase I clinical study, for example, the results showed that carlumab was well tolerated in patients with malignancy and exhibited some tumoricidal effects (99). However, a phase II clinical study in metastatic castration-resistant prostate cancer (mCRPC) revealed that carlumab did not restrain the CCL2/CCR2 pathway or exert antitumor effects, although the drug was also well tolerated in patients (100). The failure to restrict mCRPC progression suggests that blocking the CCL2/ CCR2 axis in combination with other treatments might provide better anticancer effects. Indeed, overwhelming evidence supports this hypothesis. For example, in a study of pancreatic malignancy, it was shown that the CCR2 inhibitor PF-04136309 combined with FOLFIRINOX chemotherapy could achieve an objective tumor 


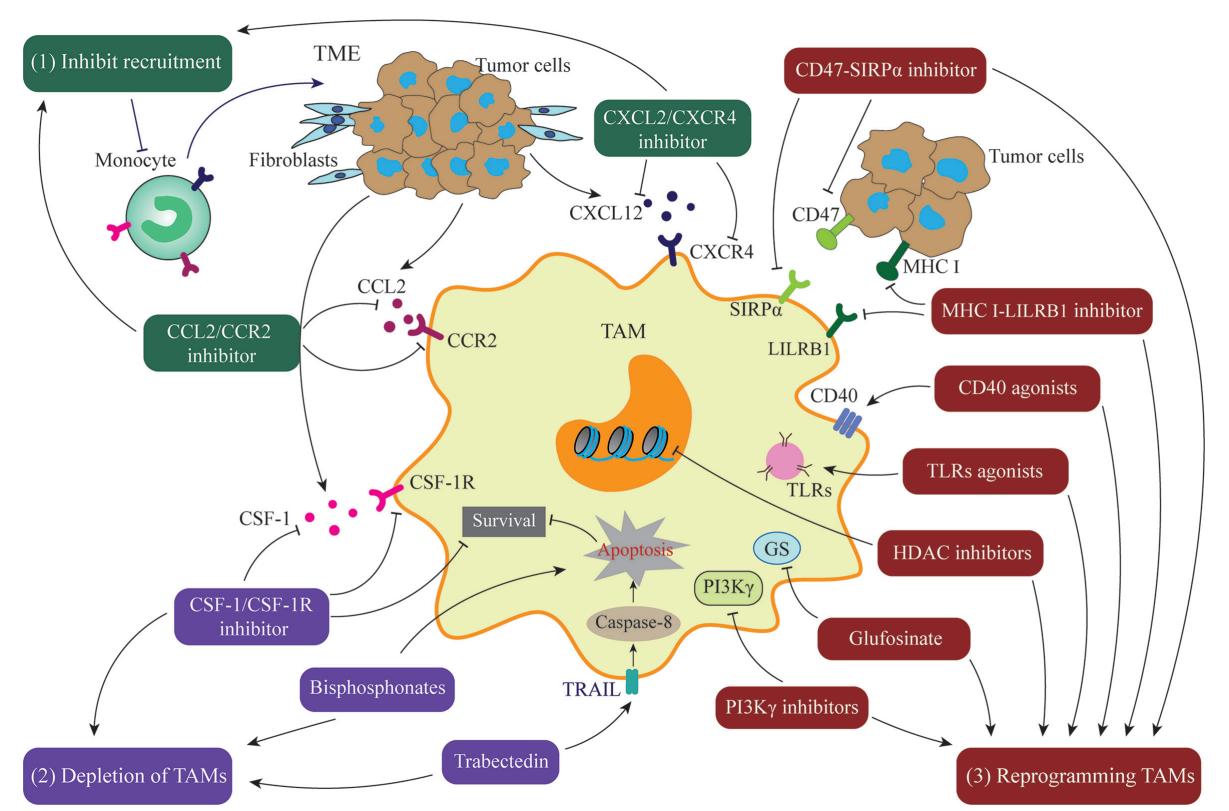

FIGURE 3 | Treatment Strategies targeting TAMs. (1) Inhibition of TAM recruitment by blocking the CCL2/CCR2 or CXCL2/CXCR4 axis. (2) Depletion of TAMs by blocking the CSF-1/CSF-1R axis or using compounds such as bisphosphonates or trabectedin. (3) Reprogramming of TAMs to activate their anti-cancer function by CD47/STRP $\alpha$ pathway inhibitors, MHC I/LILRB1 pathway inhibitors, CD40 agonists, toll-like receptors agonists, Glufosinate, PI3K $\gamma$ inhibitors, or HDAC inhibitors.

response and was safe and well tolerated (101). Furthermore, several studies have shown that the CXCL12/CXCR4 axis induces monocytes into the TME, which furthers tumorigenesis, and blockade of this axis can hinder TAM recruitment and accumulation (102-104). For instance, a study revealed that the CXCR4 antagonist, BL-8040, inhibits neuroblastoma tumors and has therapeutic potential in pediatric cancer (105). Another study indicated that the CXCL12 peptide antagonist, CTCE-99088, markedly slows tumor growth and reduces distant metastases in breast cancer (106). In addition, it was showed that chemokine CX3CL1 interacts with its receptor CX3CR1 to induce TAM recruitment into tumor tissues and promote skin cancer progression (107). Therefore, CX3CL1/CX3CR1-mediated signaling may be a new potential target for therapies that inhibit TAM recruitment into the tumor microenvironment.

In addition to elimination of TAMs by blocking macrophage recruitment, depletion of TAMs, e.g., by induction of TAM apoptosis, has also received much attention. Colonystimulating factor 1 receptor (CSF-1R), a member of the tyrosine kinase receptor family, binds to its ligands CSF-1 or IL-34 and triggers homodimerization of the receptor and subsequent activation of receptor signaling (107). CSF-1/CSF$1 \mathrm{R}$-mediated signaling plays a critical role in the survival, differentiation and maturation of macrophages (16). Numerous studies have reported that high expression of CSF-1 or its receptor CSF-1R is associated with poor prognosis in malignant tumors, such as in breast cancer and Hodgkin's lymphoma (62, 97, 108). Furthermore, CSF-1/CSF-1R signaling contributes to the conversion of TAMs from a tumor-suppressor phenotype to a tumor-promoter phenotype (109). Thus, blocking CSF-1 and CSF-1R-mediated signaling can be a promising strategy for cancer immunotherapy. Currently, several small molecules aimed at CSF-1/CSF-1R are being tested in clinical studies, including PLX3397/Pexidartinib, DCC-3014, BLZ945, FPA008/Cabiralizumab, and MCS110 (110). For instance, a study showed that PLX3397, an antagonist of CSF-1R, inhibited cell proliferation in gliomas and induced tumor regression via depletion of TAMs (111). Another study indicated that after PLX3397 treatment in mice with breast cancer, there was a significant depletion of macrophages in tumor tissues accompanied by an increase in the ratio of $\mathrm{CD}^{+} / \mathrm{CD}^{+} \mathrm{T}$ cells and significantly reduced tumor growth (112). Moreover, it was demonstrated that inhibition of CSF-1R can improve the prognosis of cancer patients $(109,112)$. Tenosynovial giant cell tumors (TGCTs), for example, are a rare and locally aggressive type of tumor characterized by high levels of CSF-1 and overexpression of CSF- $1 \mathrm{R}^{+}$macrophages (113, 114). In a clinical phase III trial for this disease, patients treated with pexidartinib showed significant improvement in symptoms and functional outcomes, and thus pexidartinib could be a potential treatment for patients with TGCT (114). However, a clinical phase II study in recurrent glioblastoma indicated that CSF-1R inhibition with PLX3397 did not significantly improve progression free survival (PFS) and failed to increase overall survival, although the agent avidly crossed the blood-tumor barrier and was well tolerated in patients (115). Other clinical data did not show significant antitumor activity of CSF1R inhibitors against relapsed or refractory classical Hodgkin lymphoma (116), implying limited clinical efficacy of targeting CSF-1 or CSF-1R and leading some companies to discontinue 
TABLE 1 | Examples of agents that target TAMs in clinical trials.

\begin{tabular}{|c|c|c|c|c|c|}
\hline Treatment strategy & Agent name & Target & Drug type & Phase & Clinical trial number \\
\hline \multirow[t]{12}{*}{ Inhibit TAMs recruitment } & Carlumab & CCL2 & CCL2 antibody & $\|$ & NCT00992186 \\
\hline & & & & । & NCT00537368 \\
\hline & & & & $\mathrm{lb}$ & NCT01204996 \\
\hline & MLN1202 & CCR2 & CCR2 antagonist & $\|$ & NCT01015560 \\
\hline & PF-04136309 & CCR2 & CCR2 antagonist & $\mathrm{lb}$ & NCT01413022 \\
\hline & AZD5069 & CCR2 & CCR2 antagonist & $1 / \|$ & NCT03177187 \\
\hline & CCX872-B & CCR2 & CCR2 antagonist & $\mathrm{lb}$ & NCT02345408 \\
\hline & Ulocuplumab & CXCR4 & CXCR4 antibody & I & NCT01120457 \\
\hline & LY2510924 & CXCR4 & CXCR4 antibody & I & NCT02737072 \\
\hline & PTX-9908 & CXCR4 & CXCR4 antagonist & $|/| \mid$ & NCT03812874 \\
\hline & Motixafortide & CXCR4 & CXCR4 antagonist & $\mathrm{l} / \mathrm{lla}$ & NCT01010880 \\
\hline & & & & Illb & NCT02907099 \\
\hline \multirow[t]{13}{*}{ Depletion of TAMs } & MCS110 & CSF-1 & CSF-1 antibody & $\mathrm{lb} / \mathrm{ll}$ & NCT02807844 \\
\hline & & & & $\|$ & NCT03785496 \\
\hline & PLX3397 & CSF-1R & CSF-1R inhibitor & 1 & NCT02777710 \\
\hline & & & & $\mid \mathrm{lb} / \mathrm{I}$ & NCT01596751 \\
\hline & & & & III & NCT02371369 \\
\hline & DCC-3014 & CSF-1R & CSF-1R inhibitor & $|/| \mid$ & NCT03069469 \\
\hline & BLZ945 & CSF-1R & CSF-1R inhibitor & $|/| \mid$ & NCT02829723 \\
\hline & FPA008 & CSF-1R & CSF-1R antibody & I & NCT03158272 \\
\hline & & & & $\|$ & NCT02471716 \\
\hline & Bisphosphonates & NA & Small molecule & III & NCT00127205 \\
\hline & & & & $\|$ & NCT00091832 \\
\hline & Trabectedin & Caspase 8 & Small molecule & I & NCT03985722 \\
\hline & & & & $\|$ & NCT02194231 \\
\hline \multirow[t]{13}{*}{ Reprogramming TAMs } & Hu5F9-G4 & CD47 & CD47 antibody & 1 & NCT02953782 \\
\hline & CC-95251 & $\operatorname{SIRP} \alpha$ & SIRP antibody & 1 & NCT03783403 \\
\hline & RO7009789 & CD40 & CD40 agonist & I & NCT02665416 \\
\hline & SEA-CD40 & CD40 & CD40 agonist & 1 & NCT02376699 \\
\hline & CP-870893 & CD40 & CD40 agonist & 1 & NCT01103635 \\
\hline & GSK1795091 & TLR4 & TLR agonist & 1 & NCT03447314 \\
\hline & IMO-2125 & TLR7/8 & TLR agonist & 1 & NCT03052205 \\
\hline & & & & $|/| \mid$ & NCT02644967 \\
\hline & & & & III & NCT03445533 \\
\hline & CMP-001 & TLR9 & TLR agonist & $\|$ & NCT03618641 \\
\hline & IPI-549 & $\mathrm{PI} 3 \mathrm{~K} \gamma$ & PI3K $\gamma$ inhibitor & $\mathrm{lb}$ & NCT02637531 \\
\hline & YY-20394 & $\mathrm{PI} 3 \mathrm{~K} \gamma$ & PI3K $\gamma$ inhibitor & 1 & NCT03757000 \\
\hline & Chidamide & HDAC & HDAC inhibitor & $\|$ & NCT04512534 \\
\hline
\end{tabular}

their myeloid targeting programs. It was observed that infiltration of MDSCs in tumor tissues increased after CSF-1R blockade, which may explain, to some extent, the limitations of the clinical efficacy of CSF-1 or CSF-1R inhibitors (117). Collectively, blockade of the CSF-1/CSF-1R axis may be a potential strategy in cancer therapy, especially for TGCTs. Nevertheless, it is increasingly clear that selecting the right patient based on tumor type, in combination with other appropriate treatments, may provide clinical benefit to cancer patients.

In addition, numerous studies have reported that some compounds, such as bisphosphonates and trabectedin, effectively remove macrophages from the tumor microenvironment by inducing apoptosis of macrophages $(118,119)$. Bisphosphonates are structurally stable, similar to pyrophosphatase, and can interact with osteoclasts to prevent bone resorption and bone metastasis (120). In breast tumors, for instance, it was observed that pyrophosphatase could be taken up by TAMs, in which it interferes with multiple functions, including polarization and survival, to exert anti-neoplastic effects (121). Trabectedin, an antineoplastic drug, could induce death of monocytes/ macrophages via a TNF-related apoptosis-inducing ligand (TRAIL)-mediated mechanism (122). A study indicated that trabectedin restricts melanoma growth and metastasis via reducing the number of TAMs in the tumor microenvironment (123).

\section{Activating Anti-Tumor TAMs}

Although a plethora of preclinical and clinical studies have revealed that elimination of TAMs can be an effective tumor treatment, we hypothesize that not all macrophages will be depleted. In addition, pro-inflammatory and tumoricidal macrophages will still be present in the TME, and if all macrophages were eliminated, the organism would potentially be at risk when encountering infectious diseases. Moreover, macrophages are well known for their plasticity in which they acquire various phenotypes, such as proinflammatory and antiinflammatory types, in different microenvironments. Thus, reprogramming TAMs to activate pro-inflammatory, anti- 
tumor properties may lead to more effective cancer treatments. In the following we summarize strategies for "re-educating" TAMs to exert anti-neoplastic effects.

Myeloid cells, such as dendritic cells and macrophages, express signal-regulated protein alpha $(\operatorname{SIRP} \alpha)$, a protein that recognizes and binds to CD47 molecules (124). CD47 is expressed on both normal and cancer cells, and interacts with SIRP $\alpha$ expressed on macrophages to send a "don't eat me" signal, thus avoiding phagocytosis by macrophages (125). Hence, it is possible to restore TAM recognition and phagocytosis of tumor cells and to activate anti-neoplastic immune responses by interfering with the SIRP $\alpha$-CD47 axis (e.g., by using antibodies to SIRP $\alpha$ and CD47) (126). This strategy was used in a study of glioblastoma, where it was observed that a CD47 monoclonal antibody converted tumor-promoting TAMs to an anti-tumor phenotype that exhibited enhanced phagocytosis of cancer cells (127). Another study found that antibody blockade of CD47 significantly increased phagocytosis of hepatocellular carcinoma cells by macrophages and promoted infiltration of proinflammatory macrophages into tumor tissue to further eliminate tumor cells (128). Moreover, it was shown that the humanized anti-CD47 antibody Hu5F9-G4 could block recognition and interaction of CD47-SIRP $\alpha$, but only slightly inhibited the activity of normal human neuronal cells (129). Therefore Hu5F9-G4 is promising for the safe treatment of malignant pediatric brain tumors.

Moreover, recent evidences have indicated that there are other "don't eat me" signals, such as the MHC class I-LILRB1 axis $(90,130,131)$. LILRB, a subfamily of leukocyte immunoglobulin-like receptor (LILR) family, contains five members (LILRB1-5), is a class of inhibitory receptors with intracellular immunoreceptor tyrosine-based inhibitory motifs (ITIM) (132). MHC class I is expressed in many nucleated cells including tumor cells, and its $\beta 2$-microglobulin subunit interacts with LILRB1, which is wildly expressed on monocytes and TAMs, thereby protecting cancer cells from being engulfed by macrophages $(90,130)$. Furthermore, blocking both CD47 and MHC I produces a synergistic effect and a stronger tumor suppression, which indicate CD47 and MHC I signals may work cooperatively (90). However, antitumor activity of cytotoxic T cells depends on the antigen presentation of MHC. Thus, strategies that specifically block $\beta 2$-microglobulin or LILRB1 seem to be more promising. LILRB2, another bestcharacterized member of LILRB family, is also mostly expressed on macrophages, monocytes and $\mathrm{CD}^{+} \mathrm{T}$ cells (131). It was suggested that LILRB2 exerted a critical role in the maturation of macrophages; antagonism of LILRB2 with specific monoclonal antibodies could impair the inhibitory effect of macrophages on $\mathrm{T}$ cell proliferation, reduce the infiltration of MDSCs and Tregs in tumor tissue, enhance the phagocytosis by macrophages, reprogram TAMs to a proinflammatory phenotype and promote antitumor immunity (133). Additionally, recent study reported that blockade of LILRB4 could convert anti-inflammatory macrophages to a more inflammatory phenotype, which may be another potential target for therapies that activate anti-cancer function of TAMs (134). However, these mechanisms need to be further confirmed and validated in more clinical trials.

In addition to blocking "don't eat me" signals, there are other strategies that target TAMs, such as CD40 agonists, toll-like receptor agonists, $\mathrm{PI} 3 \mathrm{~K} \gamma$ inhibitors, and histone deacetylase (HDAC) inhibitors $(10,16)$. CD40, a molecule of the TNF receptor superfamily, is expressed on macrophages and other antigen-presenting cells (APCs). Once activated by its ligand CD40L, CD40 promotes the expression of MHC molecules and stimulates the release of pro-inflammatory cytokines from APCs, which exert anti-neoplastic effects by supporting effector T-cells (135). In a study of pancreatic ductal adenocarcinoma, for example, it was observed that a CD40 agonist prompted macrophage infiltration into tumor tissue and converted TAMs to an anti-tumor phenotype, and a combination of CD40 agonists and the chemotherapeutic agent gemcitabine resulted in tumor remission in patients with advanced disease (136). In addition, it has been shown that CD40 agonists exhibit more potent antitumor efficacy when combined with mitogenactivated protein kinase inhibitors (MEKi) by enhancing tumoricidal immune responses and attenuating the immunosuppressive cell activities of M2 macrophages, Tregs and MDSCs (137).

Toll-like receptors (TLRs) are an evolutionarily ancient family of pattern recognition receptors that play a key role in activating the innate immune response; TLRs can induce macrophage differentiation into a pro-inflammatory phenotype upon activation by viral nucleic acids (i.e., DNA or RNA) or bacterial particles (e.g., lipopolysaccharides) (138). Based on this property of TLRs, TLR agonists, such as TLR4, TLR7/8 and TLR9, are currently tested in cancer research with some success in stimulating TAM polarization into an anti-tumor phenotype (139). For instance, it was observed that the TLR7/8 agonist can induce MDSCs to differentiate into tumoricidal macrophages, which makes it a potential agent for treatment of oxaliplatinresistant colorectal carcinoma (140). Notably, bacilli calmette guerin (BCG), the first FDA-approved TLR-agonist for the treatment of bladder cancer, stimulates TLR2 and TLR4, which convert TAMs toward anti-tumor types and enhance the cytotoxicity of macrophages against neoplastic cells (141). Furthermore, intratumoral administration of TLR-stimulating drugs has not only shown effective local antitumor efficacy, but also reduces distant tumor metastasis by activating the systemic immune system (142). Additionally, TLR7/8 agonist MEDI9197 combined with immune checkpoint inhibitors PD1 blockade can polarize TAMs toward anti-tumor phenotypes and activate $\mathrm{CD}^{+} \mathrm{T}$ cells and NK cells, leading to a better efficacy (143).

In addition, a number of kinase, metabolic or epigenetic enzyme inhibitors are being tested in vitro and in vivo to control the polarization and activation of macrophages (144). For example, PI3K $\gamma$ is widely expressed on macrophages and other myeloid cells, and is the only class 1B PI3K member (145). Activation of PI3K $\gamma$ signaling can trigger an immunosuppressive transcriptional profile of TAMs and promote cancer progression (146). As a result, PI3K $\gamma$ inactivation induces tumor regression 
via up-regulation of pro-inflammatory cytokines such as IL-12 and stimulation of $\mathrm{CD}^{+} \mathrm{T}$ cells (146). Glutamine not only plays a critical role in numerous biological functions (e.g., amino acid production, nucleotide synthesis, and extracellular matrix production), but also promotes tumor growth and creates an immunosuppressive TME (147). However, Inhibition of glutamine synthetase (GS) with an antagonist, glufosinate, can impair cancer metastasis in several mouse tumor models, which is associated with blockade of immunosuppression and angiogenesis and re-education of TAMs to an anti-neoplastic type (148). Additionally, studies have reported that epigenetic remodeling of TAMs, such as inhibition of histone deacetylase (HDAC), promotes repolarization of macrophages to an antitumor phenotype and activates $\mathrm{T}$ cell responses (149, 150). Moreover, research has shown that TMP195, an antagonist of HDAC, reduced tumor growth and metastasis by modulating TAMs into a tumoricidal phenotype; furthermore, TMP195 enhanced the antitumor effect of PD-1 treatment and chemotherapy (150). Collectively, these results demonstrate that reprogramming TAMs to anti-tumorigenic macrophages is a promising strategy that has the potential to improve the prognosis of cancer patients.

As mentioned above, the manipulation of TAMs to exert antineoplastic effects has been investigated using different therapeutic approaches, such as CD47-SIRP1a or MHC ILILRB1inhibitors can restore the ability of TAMs to engulf tumor cells, TLRs and CD40 agonists and other inhibitors can stimulate TAMs to become tumoricidal effector cells. All of these reprogramming strategies are based on activated macrophages that are either directly cytotoxic to tumor cells or indirectly activate cytotoxic T cells or NK cells. However, there is evidence suggests that anti-tumor TAMs are required for efficacy of therapy response. In a study of pancreatic ductal adenocarcinoma, patients with substantial infiltration of TAMs at the tumor site responded better to gemcitabine; further analysis indicates that TAMs exposed to gemcitabine were converted to antitumor types with activation of cytotoxicity genes (151). Similarly, paclitaxel, a chemotherapeutic drug, is showed to rewire TAMs toward tumoricidal phenotypes through activation of TLR4 on TAMs (152). Another clinical phase II trial in metastatic gastric cancer suggested that high infiltration of M2 macrophages in the TME may enhance the sensitivity of cabazitaxel (a novel taxoid) and improve survival (153). The mechanism by which TAMs enhance the efficacy of chemotherapy may be related to the immunogenic cell death (ICD) of neoplastic cells caused by chemotherapeutic drugs (154). When tumor cells die through ICD, they may emit a large number of molecules, such as damage-associated molecular patterns (DAMP), cytokines, tumor antigens and immunostimulatory signals, which ultimately switch TAMs toward tumoricidal phenotypes and activate adaptive immune responses (155). Other therapies such as radiotherapy, and cancer vaccines, may also require TAMs to enhance efficacy $(156,157)$. In addition to being activated by ICD death of tumor cells, TAMs can also be induced and activated by cytotoxic $\mathrm{T}$ cells to exert anti-tumor functions after therapy. In turn, tumoricidal TAMs possess the ability to support effector $\mathrm{T}$ cells. For example, in a model of cervical carcinoma, synthetic long peptide (SLP) vaccines can induce tumor regression not only by inducing cytotoxic CD8+ T cells to infiltrate tumor sites, but also by attracting macrophages by these vaccine-induced $\mathrm{T}$ cells; depletion of TAMs with CSF-1R inhibitor abrogates the complete tumor remission elicited by SLP therapy (157). Another study suggests that specific depletion of the CD163+ macrophages can trigger tumor shrinkage in a melanoma model by increasing infiltration of effector $\mathrm{T}$ cells and concomitant recruitment of pro-inflammatory TAMs (158). Likewise, it is implied that IL-1 $\beta$ contributes to an immunosuppressive TME and favors tumor growth; inhibition of IL1 $\beta$ drives the accumulation of $\mathrm{CD}^{+} \mathrm{T}$ cells at tumor sites, which subsequently activates TAMs and induces tumor regression; nevertheless, depletion of TAMs with CSF-1R inhibitor can abolish tumor shrinkage caused by IL- $1 \beta$ deficient (159). These findings suggest that macrophage-T cell cross-talk plays an important role in the anti-tumor immune response. However, more research is needed to better understand these mechanisms.

\section{CONCLUSION}

TAMs, an important class of innate immune cells in the tumor microenvironment, are widely expressed in a variety of tumor tissues, while massive infiltration of TAMs or enrichment of TAM-related genes usually indicates tumor progression or poor disease prognosis. Moreover, studies have overwhelmingly shown that TAMs are intimately involved in multiple tumorrelated processes by contributing to tumorigenesis and proliferation, accelerating angiogenesis, promoting invasion and metastasis, inducing cancer stem cell formation, triggering treatment resistance, and immunosuppression. Thus, the various tumor-promoting mechanisms of TAMs exhibit numerous appealing targets for cancer treatment. Tumor immunotherapies targeting TAMs, including inhibition of TAM recruitment, acceleration of TAM depletion or apoptosis, activation of phagocytosis by TAMs, and modulation of TAM anti-tumor polarization, have shown great potential in preclinical and clinical cancer research. It is encouraging to see that drugs targeting TAMs are in clinical trials and showing antitumor effects. Collectively, strategies that target TAMs may well turn out to be promising in tumor immunotherapy.

Nevertheless, there are some issues and limitations that remain to be addressed. Firstly, TRM-derived TAMs and monocyte-derived TAMs are both present in the TME. Recruitment of monocyte-derived TAMs can be achieved by inhibition of trafficking receptors (e.g., CC2R), but TRMs do not seem to be recruited in this way. Thus, strategies that can differentially block the recruitment of TRMs are needed. Secondly, non-specific depletion of TAMs results in some tumoricidal macrophages also being eliminated. Therefore, the long-term consequences of TAM depletion are still unclear. Finally, repolarization of TAMs towards an anti-tumorigenic phenotype may trigger excessive macrophage activation and thus be associated with substantial toxicity, such as macrophage 
activation syndrome and hemophagocytic syndrome. Therefore, more research is needed to deepen the understanding of TAMs.

\section{AUTHOR CONTRIBUTIONS}

YT conceived and designed the figure, as well as wrote and revised the manuscript. MW collected the related reference and revised the manuscript. YZ and SG edited the manuscript. FZ, GX, and CS provided guidance and revised the manuscript.

\section{REFERENCES}

1. Ferlay J, Soerjomataram I, Dikshit R, Eser S, Mathers C, Rebelo M, et al. Cancer Incidence and Mortality Worldwide: Sources, Methods and Major Patterns in GLOBOCAN 2012. Int J Cancer (2015) 136:E359-86. doi: $10.1002 /$ ijc. 29210

2. Siegel RL, Miller KD, Jemal A. Cancer Statistics, 2019. CA Cancer J Clin (2019) 69:7-34. doi: 10.3322/caac.21551

3. Miller KD, Nogueira L, Mariotto AB, Rowland JH, Yabroff KR, Alfano CM, et al. Cancer Treatment and Survivorship Statistics, 2019. CA Cancer J Clin (2019) 69:363-85. doi: 10.3322/caac.21565

4. Xiao Y, Yu D. Tumor Microenvironment as a Therapeutic Target in Cancer. Pharmacol Ther (2020) 221:107753. doi: 10.1016/j.pharmthera.2020.107753

5. Hinshaw DC, Shevde LA. The Tumor Microenvironment Innately Modulates Cancer Progression. Cancer Res (2019) 79:4557-66. doi: 10.1158/0008-5472.CAN-18-3962

6. Ginhoux F, Jung S. Monocytes and Macrophages: Developmental Pathways and Tissue Homeostasis. Nat Rev Immunol (2014) 14:392-404. doi: 10.1038/ nri3671

7. Shapouri-Moghaddam A, Mohammadian S, Vazini H, Taghadosi M, Esmaeili SA, Mardani F, et al. Macrophage Plasticity, Polarization, and Function in Health and Disease. J Cell Physiol (2018) 233:6425-40. doi: $10.1002 /$ jcp. 26429

8. Quail DF, Joyce JA. Molecular Pathways: Deciphering Mechanisms of Resistance to Macrophage-Targeted Therapies. Clin Cancer Res (2017) 23:876-84. doi: 10.1158/1078-0432.CCR-16-0133

9. Murray PJ. Macrophage Polarization. Annu Rev Physiol (2017) 79:541-66. doi: 10.1146/annurev-physiol-022516-034339

10. Cheng N, Bai X, Shu Y, Ahmad O, Shen P. Targeting Tumor-Associated Macrophages as an Antitumor Strategy. Biochem Pharmacol (2020) 183:114354. doi: 10.1016/j.bcp.2020.114354

11. Zhang H, Li R, Cao Y, Gu Y, Lin C, Liu X, et al. Poor Clinical Outcomes and Immunoevasive Contexture in Intratumoral Il-10-Producing Macrophages Enriched Gastric Cancer Patients. Ann Surg (2020). doi: 10.1097/ SLA. 0000000000004037

12. Muhitch JB, Hoffend NC, Azabdaftari G, Miller A, Bshara W, Morrison CD, et al. Tumor-Associated Macrophage Expression of Interferon Regulatory Factor-8 (IRF8) is a Predictor of Progression and Patient Survival in Renal Cell Carcinoma. J Immunother Cancer (2019) 7:155. doi: 10.1186/s40425-019-0630-0

13. Zhang WJ, Wang XH, Gao ST, Chen C, Xu XY, Sun Q, et al. Tumor-Associated Macrophages Correlate With Phenomenon of Epithelial-Mesenchymal Transition and Contribute to Poor Prognosis in Triple-Negative Breast Cancer Patients. J Surg Res (2018) 222:93-101. doi: 10.1016/j.jss.2017.09.035

14. Edin S, Wikberg ML, Oldenborg PA, Palmqvist R. Macrophages: Good Guys in Colorectal Cancer. Oncoimmunology (2013) 2:e23038. doi: 10.4161/ onci. 23038

15. El-Arabey AA, Denizli M, Kanlikilicer P, Bayraktar R, Ivan C, Rashed M, et al. GATA3 as a Master Regulator for Interactions of Tumor-Associated Macrophages With High-Grade Serous Ovarian Carcinoma. Cell Signal (2020) 68:109539. doi: 10.1016/j.cellsig.2020.109539

16. Mantovani A, Marchesi F, Malesci A, Laghi L, Allavena P. TumourAssociated Macrophages as Treatment Targets in Oncology. Nat Rev Clin Oncol (2017) 14:399-416. doi: 10.1038/nrclinonc.2016.217
All authors contributed to the article and approved the submitted version.

\section{FUNDING}

This work was supported by the National Key Research and Development Program of China (2018YFC0910700, 2016YFB0201702) and Chenguang Program of Shanghai Municipal Education Commission (No.158554).

17. Mantovani A, Marchesi F, Jaillon S, Garlanda C, Allavena P. TumorAssociated Myeloid Cells: Diversity and Therapeutic Targeting. Cell Mol Immunol (2021) 18:566-78. doi: 10.1038/s41423-020-00613-4

18. Guerriero JL. Macrophages: The Road Less Traveled, Changing Anticancer Therapy. Trends Mol Med (2018) 24:472-89. doi: 10.1016/j.molmed. 2018.03.006

19. Biswas SK, Allavena P, Mantovani A. Tumor-Associated Macrophages: Functional Diversity, Clinical Significance, and Open Questions. Semin Immunopathol (2013) 35:585-600. doi: 10.1007/s00281-013-0367-7

20. Gomez PE, Klapproth K, Schulz C, Busch K, Azzoni E, Crozet L, et al. Tissue-Resident Macrophages Originate From Yolk-Sac-Derived ErythroMyeloid Progenitors. Nature (2015) 518:547-51. doi: 10.1038/nature13989

21. Ginhoux F, Guilliams M. Tissue-Resident Macrophage Ontogeny and Homeostasis. Immunity (2016) 44:439-49. doi: 10.1016/j.immuni.2016. 02.024

22. Perdiguero EG, Geissmann F. The Development and Maintenance of Resident Macrophages. Nat Immunol (2016) 17:2-8. doi: 10.1038/ni.3341

23. DeNardo DG, Ruffell B. Macrophages as Regulators of Tumour Immunity and Immunotherapy. Nat Rev Immunol (2019) 19:369-82. doi: 10.1038/ s41577-019-0127-6

24. Bian Z, Gong Y, Huang T, Lee C, Bian L, Bai Z, et al. Deciphering Human Macrophage Development At Single-Cell Resolution. Nature (2020) 582:571-6. doi: 10.1038/s41586-020-2316-7

25. Ostuni R, Kratochvill F, Murray PJ, Natoli G. Macrophages and Cancer: From Mechanisms to Therapeutic Implications. Trends Immunol (2015) 36:229-39. doi: 10.1016/j.it.2015.02.004

26. Franklin RA, Liao W, Sarkar A, Kim MV, Bivona MR, Liu K, et al. The Cellular and Molecular Origin of Tumor-Associated Macrophages. Science (2014) 344:921-5. doi: 10.1126/science.1252510

27. Zhu Y, Herndon JM, Sojka DK, Kim KW, Knolhoff BL, Zuo C, et al. TissueResident Macrophages in Pancreatic Ductal Adenocarcinoma Originate From Embryonic Hematopoiesis and Promote Tumor Progression. Immunity (2017) 47:323-38. doi: 10.1016/j.immuni.2017.07.014

28. Loyher PL, Hamon P, Laviron M, Meghraoui-Kheddar A, Goncalves E, Deng Z, et al. Macrophages of Distinct Origins Contribute to Tumor Development in the Lung. J Exp Med (2018) 215:2536-53. doi: 10.1084/jem.20180534

29. Spooner CJ, Cheng JX, Pujadas E, Laslo P, Singh H. A Recurrent Network Involving the Transcription Factors PU.1 and Gfil Orchestrates Innate and Adaptive Immune Cell Fates. Immunity (2009) 31:576-86. doi: 10.1016/ j.immuni.2009.07.011

30. Lavin Y, Winter D, Blecher-Gonen R, David E, Keren-Shaul H, Merad M, et al. Tissue-Resident Macrophage Enhancer Landscapes are Shaped by the Local Microenvironment. Cell (2014) 159:1312-26. doi: 10.1016/ j.cell.2014.11.018

31. Chen Z, Feng X, Herting CJ, Garcia VA, Nie K, Pong WW, et al. Cellular and Molecular Identity of Tumor-Associated Macrophages in Glioblastoma. Cancer Res (2017) 77:2266-78. doi: 10.1158/0008-5472.CAN-16-2310

32. Mantovani A, Sozzani S, Locati M, Allavena P, Sica A. Macrophage Polarization: Tumor-Associated Macrophages as a Paradigm for Polarized M2 Mononuclear Phagocytes. Trends Immunol (2002) 23:549-55. doi: 10.1016/s1471-4906(02)02302-5

33. Ye YC, Zhao JL, Lu YT, Gao CC, Yang Y, Liang SQ, et al. Notch Signaling Via WNT Regulates the Proliferation of Alternative, Ccr2-Independent 
Tumor-Associated Macrophages in Hepatocellular Carcinoma. Cancer Res (2019) 79:4160-72. doi: 10.1158/0008-5472.CAN-18-1691

34. Tong Y, Zhou L, Yang L, Guo P, Cao Y, Qin FX, et al. Concomitant Type I IFN and M-CSF Signaling Reprograms Monocyte Differentiation and Drives Pro-Tumoral Arginase Production. Ebiomedicine (2019) 39:132-44. doi: 10.1016/j.ebiom.2018.11.062

35. Bashir S, Sharma Y, Elahi A, Khan F. Macrophage Polarization: The Link Between Inflammation and Related Diseases. Inflammation Res (2016) 65:111. doi: 10.1007/s00011-015-0874-1

36. Wynn TA, Vannella KM. Macrophages in Tissue Repair, Regeneration, and Fibrosis. Immunity (2016) 44:450-62. doi: 10.1016/j.immuni.2016.02.015

37. Colin S, Chinetti-Gbaguidi G, Staels B. Macrophage Phenotypes in Atherosclerosis. Immunol Rev (2014) 262:153-66. doi: 10.1111/imr.12218

38. Wang LX, Zhang SX, Wu HJ, Rong XL, Guo J. M2b Macrophage Polarization and its Roles in Diseases. J Leukoc Biol (2019) 106:345-58. doi: 10.1002/JLB.3RU1018-378RR

39. Ferrante CJ, Leibovich SJ. Regulation of Macrophage Polarization and Wound Healing. Adv Wound Care (New Rochelle) (2012) 1:10-6. doi: 10.1089/wound.2011.0307

40. Zhou J, Tang Z, Gao S, Li C, Feng Y, Zhou X. Tumor-Associated Macrophages: Recent Insights and Therapies. Front Oncol (2020) 10:188. doi: $10.3389 /$ fonc. 2020.00188

41. Zhu C, Kros JM, Cheng C, Mustafa D. The Contribution of TumorAssociated Macrophages in Glioma Neo-Angiogenesis and Implications for Anti-Angiogenic Strategies. Neuro Oncol (2017) 19:1435-46. doi: 10.1093/neuonc/nox081

42. Biswas SK, Mantovani A. Macrophage Plasticity and Interaction With Lymphocyte Subsets: Cancer as a Paradigm. Nat Immunol (2010) 11:88996. doi: 10.1038/ni.1937

43. Salmaninejad A, Valilou SF, Soltani A, Ahmadi S, Abarghan YJ, Rosengren RJ, et al. Tumor-Associated Macrophages: Role in Cancer Development and Therapeutic Implications. Cell Oncol (Dordr) (2019) 42:591-608. doi: 10.1007/s13402-019-00453-z

44. Gabrilovich DI, Ostrand-Rosenberg S, Bronte V. Coordinated Regulation of Myeloid Cells by Tumours. Nat Rev Immunol (2012) 12:253-68. doi: $10.1038 /$ nri3175

45. Pallotta MT, Orabona C, Volpi C, Vacca C, Belladonna ML, Bianchi R, et al. Indoleamine 2,3-Dioxygenase is a Signaling Protein in Long-Term Tolerance by Dendritic Cells. Nat Immunol (2011) 12:870-8. doi: 10.1038/ ni. 2077

46. Hambardzumyan D, Gutmann DH, Kettenmann H. The Role of Microglia and Macrophages in Glioma Maintenance and Progression. Nat Neurosci (2016) 19:20-7. doi: 10.1038/nn.4185

47. Weissleder R, Pittet MJ. The Expanding Landscape of Inflammatory Cells Affecting Cancer Therapy. Nat BioMed Eng (2020) 4:489-98. doi: 10.1038/ s41551-020-0524-y

48. Li C, Xu X, Wei S, Jiang P, Xue L, Wang J. Tumor-Associated Macrophages: Potential Therapeutic Strategies and Future Prospects in Cancer. J Immunother Cancer (2021) 9:e001341. doi: 10.1136/jitc-2020-001341

49. Wu K, Lin K, Li X, Yuan X, Xu P, Ni P, et al. Redefining Tumor-Associated Macrophage Subpopulations and Functions in the Tumor Microenvironment. Front Immunol (2020) 11:1731. doi: 10.3389/fimmu. 2020.01731

50. Park JY, Sung JY, Lee J, Park YK, Kim YW, Kim GY, et al. Polarized CD163+ Tumor-Associated Macrophages are Associated With Increased Angiogenesis and CXCL12 Expression in Gastric Cancer. Clin Res Hepatol Gastroenterol (2016) 40:357-65. doi: 10.1016/j.clinre.2015.09.005

51. Crusz SM, Balkwill FR. Inflammation and Cancer: Advances and New Agents. Nat Rev Clin Oncol (2015) 12:584-96. doi: 10.1038/ nrclinonc.2015.105

52. Morrison WB. Inflammation and Cancer: A Comparative View. J Vet Intern Med (2012) 26:18-31. doi: 10.1111/j.1939-1676.2011.00836.x

53. Shacter E, Weitzman SA. Chronic Inflammation and Cancer. Oncol (Williston Park) (2002) 16:217-26, 229, 230-2.

54. Moghaddam SJ, Li H, Cho SN, Dishop MK, Wistuba II, Ji L, et al. Promotion of Lung Carcinogenesis by Chronic Obstructive Pulmonary Disease-Like Airway Inflammation in a K-ras-Induced Mouse Model. Am J Respir Cell Mol Biol (2009) 40:443-53. doi: 10.1165/rcmb.2008-0198OC
55. Coussens LM, Werb Z. Inflammation and Cancer. Nature (2002) 420:860-7. doi: 10.1038/nature01322

56. Deng L, Zhou JF, Sellers RS, Li JF, Nguyen AV, Wang Y, et al. A Novel Mouse Model of Inflammatory Bowel Disease Links Mammalian Target of Rapamycin-Dependent Hyperproliferation of Colonic Epithelium to Inflammation-Associated Tumorigenesis. Am J Pathol (2010) 176:952-67. doi: 10.2353/ajpath.2010.090622

57. Oft M. Il-10: Master Switch From Tumor-Promoting Inflammation to Antitumor Immunity. Cancer Immunol Res (2014) 2:194-9. doi: 10.1158/ 2326-6066.CIR-13-0214

58. Canli O, Nicolas AM, Gupta J, Finkelmeier F, Goncharova O, Pesic M, et al. Myeloid Cell-Derived Reactive Oxygen Species Induce Epithelial Mutagenesis. Cancer Cell (2017) 32:869-83. doi: 10.1016/j.ccell.2017.11.004

59. Wang B, Li Q, Qin L, Zhao S, Wang J, Chen X. Transition of TumorAssociated Macrophages From MHC Class II(hi) to MHC Class II(low) Mediates Tumor Progression in Mice. BMC Immunol (2011) 12:43. doi: 10.1186/1471-2172-12-43

60. Vlaicu P, Mertins P, Mayr T, Widschwendter P, Ataseven B, Hogel B, et al. Monocytes/Macrophages Support Mammary Tumor Invasivity by CoSecreting Lineage-Specific EGFR Ligands and a STAT3 Activator. BMC Cancer (2013) 13:197. doi: 10.1186/1471-2407-13-197

61. Giurisato E, Xu Q, Lonardi S, Telfer B, Russo I, Pearson A, et al. Myeloid ERK5 Deficiency Suppresses Tumor Growth by Blocking Protumor Macrophage Polarization Via STAT3 Inhibition. Proc Natl Acad Sci USA (2018) 115:E2801-10. doi: 10.1073/pnas.1707929115

62. Lin EY, Nguyen AV, Russell RG, Pollard JW. Colony-Stimulating Factor 1 Promotes Progression of Mammary Tumors to Malignancy. J Exp Med (2001) 193:727-40. doi: 10.1084/jem.193.6.727

63. Donnem T, Reynolds AR, Kuczynski EA, Gatter K, Vermeulen PB, Kerbel RS, et al. Non-Angiogenic Tumours and Their Influence on Cancer Biology. Nat Rev Cancer (2018) 18:323-36. doi: 10.1038/nrc.2018.14

64. De Palma M, Biziato D, Petrova TV. Microenvironmental Regulation of Tumour Angiogenesis. Nat Rev Cancer (2017) 17:457-74. doi: 10.1038/ nrc.2017.51

65. Riabov V, Gudima A, Wang N, Mickley A, Orekhov A, Kzhyshkowska J. Role of Tumor Associated Macrophages in Tumor Angiogenesis and Lymphangiogenesis. Front Physiol (2014) 5:75. doi: 10.3389/fphys. 2014.00075

66. Lin L, Chen YS, Yao YD, Chen JQ, Chen JN, Huang SY, et al. CCL18 From Tumor-Associated Macrophages Promotes Angiogenesis in Breast Cancer. Oncotarget (2015) 6:34758-73. doi: 10.18632/oncotarget.5325

67. Ruffell B, Coussens LM. Macrophages and Therapeutic Resistance in Cancer. Cancer Cell (2015) 27:462-72. doi: 10.1016/j.ccell.2015.02.015

68. Viallard C, Larrivee B. Tumor Angiogenesis and Vascular Normalization: Alternative Therapeutic Targets. Angiogenesis (2017) 20:409-26. doi: 10.1007/s10456-017-9562-9

69. Yeo EJ, Cassetta L, Qian BZ, Lewkowich I, Li JF, Stefater JR, et al. Myeloid WNT7b Mediates the Angiogenic Switch and Metastasis in Breast Cancer. Cancer Res (2014) 74:2962-73. doi: 10.1158/0008-5472.CAN-13-2421

70. Kawahara A, Hattori S, Akiba J, Nakashima K, Taira T, Watari K, et al. Infiltration of Thymidine Phosphorylase-Positive Macrophages is Closely Associated With Tumor Angiogenesis and Survival in Intestinal Type Gastric Cancer. Oncol Rep (2010) 24:405-15. doi: 10.3892/or_00000873

71. Steeg PS. Targeting Metastasis. Nat Rev Cancer (2016) 16:201-18. doi: $10.1038 / \mathrm{nrc} .2016 .25$

72. Lin $\mathrm{Y}, \mathrm{Xu}$ J, Lan H. Tumor-Associated Macrophages in Tumor Metastasis: Biological Roles and Clinical Therapeutic Applications. J Hematol Oncol (2019) 12:76. doi: 10.1186/s13045-019-0760-3

73. Wei C, Yang C, Wang S, Shi D, Zhang C, Lin X, et al. Crosstalk Between Cancer Cells and Tumor Associated Macrophages Is Required for Mesenchymal Circulating Tumor Cell-Mediated Colorectal Cancer Metastasis. Mol Cancer (2019) 18:64. doi: 10.1186/s12943-019-0976-4

74. Harney AS, Arwert EN, Entenberg D, Wang Y, Guo P, Qian BZ, et al. RealTime Imaging Reveals Local, Transient Vascular Permeability, and Tumor Cell Intravasation Stimulated by TIE2hi Macrophage-Derived Vegfa. Cancer Discovery (2015) 5:932-43. doi: 10.1158/2159-8290.CD-15-0012

75. Zhao L, Lim SY, Gordon-Weeks AN, Tapmeier TT, Im JH, Cao Y, et al. Recruitment of a Myeloid Cell Subset (CD11b/Gr1 Mid) Via CCL2/CCR2 
Promotes the Development of Colorectal Cancer Liver Metastasis. Hepatology (2013) 57:829-39. doi: 10.1002/hep.26094

76. Kitamura T, Qian BZ, Soong D, Cassetta L, Noy R, Sugano G, et al. CCL2Induced Chemokine Cascade Promotes Breast Cancer Metastasis by Enhancing Retention of Metastasis-Associated Macrophages. J Exp Med (2015) 212:1043-59. doi: 10.1084/jem.20141836

77. Kitamura T, Doughty-Shenton D, Cassetta L, Fragkogianni S, Brownlie D, Kato Y, et al. Monocytes Differentiate to Immune Suppressive Precursors of Metastasis-Associated Macrophages in Mouse Models of Metastatic Breast Cancer. Front Immunol (2017) 8:2004. doi: 10.3389/fimmu.2017.02004

78. Li X, Yao W, Yuan Y, Chen P, Li B, Li J, et al. Targeting of TumourInfiltrating Macrophages Via CCL2/CCR2 Signalling as a Therapeutic Strategy Against Hepatocellular Carcinoma. Gut (2017) 66:157-67. doi: 10.1136/gutjnl-2015-310514

79. Chen Q, Zhang XH, Massague J. Macrophage Binding to Receptor VCAM-1 Transmits Survival Signals in Breast Cancer Cells That Invade the Lungs. Cancer Cell (2011) 20:538-49. doi: 10.1016/j.ccr.2011.08.025

80. Komohara Y, Fujiwara Y, Ohnishi K, Takeya M. Tumor-Associated Macrophages: Potential Therapeutic Targets for Anti-Cancer Therapy. Adv Drug Delivery Rev (2016) 99:180-5. doi: 10.1016/j.addr.2015.11.009

81. Chen EP, Markosyan N, Connolly E, Lawson JA, Li X, Grant GR, et al. Myeloid Cell COX-2 Deletion Reduces Mammary Tumor Growth Through Enhanced Cytotoxic T-lymphocyte Function. Carcinogenesis (2014) 35:1788-97. doi: 10.1093/carcin/bgu053

82. Chang AL, Miska J, Wainwright DA, Dey M, Rivetta CV, Yu D, et al. Ccl2 Produced by the Glioma Microenvironment Is Essential for the Recruitment of Regulatory T Cells and Myeloid-Derived Suppressor Cells. Cancer Res (2016) 76:5671-82. doi: 10.1158/0008-5472.CAN-16-0144

83. Liu J, Zhang N, Li Q, Zhang W, Ke F, Leng Q, et al. Tumor-Associated Macrophages Recruit CCR6+ Regulatory T Cells and Promote the Development of Colorectal Cancer Via Enhancing CCL20 Production in Mice. PloS One (2011) 6:e19495. doi: 10.1371/journal.pone.0019495

84. Prima V, Kaliberova LN, Kaliberov S, Curiel DT, Kusmartsev S. Cox2/ mPGES1/PGE2 Pathway Regulates PD-L1 Expression in Tumor-Associated Macrophages and Myeloid-Derived Suppressor Cells. Proc Natl Acad Sci USA (2017) 114:1117-22. doi: 10.1073/pnas.1612920114

85. Lee YH, Martin-Orozco N, Zheng P, Li J, Zhang P, Tan H, et al. Inhibition of the B7-H3 Immune Checkpoint Limits Tumor Growth by Enhancing Cytotoxic Lymphocyte Function. Cell Res (2017) 27:1034-45. doi: 10.1038/ cr.2017.90

86. Li J, Lee Y, Li Y, Jiang Y, Lu H, Zang W, et al. Co-Inhibitory Molecule B7 Superfamily Member 1 Expressed by Tumor-Infiltrating Myeloid Cells Induces Dysfunction of Anti-tumor Cd8(+) T Cells. Immunity (2018) 48:773-86. doi: 10.1016/j.immuni.2018.03.018

87. Rodriguez PC, Zea AH, DeSalvo J, Culotta KS, Zabaleta J, Quiceno DG, et al. L-Arginine Consumption by Macrophages Modulates the Expression of CD3 Zeta Chain in T Lymphocytes. J Immunol (2003) 171:1232-9. doi: 10.4049/jimmunol.171.3.1232

88. Geiger R, Rieckmann JC, Wolf T, Basso C, Feng Y, Fuhrer T, et al. LArginine Modulates T Cell Metabolism and Enhances Survival and AntiTumor Activity. Cell (2016) 167:829-42. doi: 10.1016/j.cell.2016.09.031

89. Morandi F, Pistoia V. Interactions Between HLA-G and HLA-E in Physiological and Pathological Conditions. Front Immunol (2014) 5:394. doi: 10.3389/fimmu.2014.00394

90. Barkal AA, Weiskopf K, Kao KS, Gordon SR, Rosental B, Yiu YY, et al. Engagement of MHC Class I by the Inhibitory Receptor LILRB1 Suppresses Macrophages and is a Target of Cancer Immunotherapy. Nat Immunol (2018) 19:76-84. doi: 10.1038/s41590-017-0004-Z

91. Yang L, Shi P, Zhao G, Xu J, Peng W, Zhang J, et al. Targeting Cancer Stem Cell Pathways for Cancer Therapy. Signal Transduct Target Ther (2020) 5:8. doi: 10.1038/s41392-020-0110-5

92. Batlle E, Clevers H. Cancer Stem Cells Revisited. Nat Med (2017) 23:112434. doi: $10.1038 / \mathrm{nm} .4409$

93. Lu H, Clauser KR, Tam WL, Frose J, Ye X, Eaton EN, et al. A Breast Cancer Stem Cell Niche Supported by Juxtacrine Signalling From Monocytes and Macrophages. Nat Cell Biol (2014) 16:1105-17. doi: 10.1038/ncb3041

94. Wan S, Zhao E, Kryczek I, Vatan L, Sadovskaya A, Ludema G, et al. TumorAssociated Macrophages Produce Interleukin 6 and Signal Via STAT3 to
Promote Expansion of Human Hepatocellular Carcinoma Stem Cells. Gastroenterology (2014) 147:1393-404. doi: 10.1053/j.gastro.2014.08.039

95. Yang J, Liao D, Chen C, Liu Y, Chuang TH, Xiang R, et al. TumorAssociated Macrophages Regulate Murine Breast Cancer Stem Cells Through a Novel Paracrine EGFR/Stat3/Sox-2 Signaling Pathway. Stem Cells (2013) 31:248-58. doi: 10.1002/stem.1281

96. Li D, Ji H, Niu X, Yin L, Wang Y, Gu Y, et al. Tumor-Associated Macrophages Secrete CC-Chemokine Ligand 2 and Induce Tamoxifen Resistance by Activating PI3K/Akt/mTOR in Breast Cancer. Cancer Sci (2020) 111:47-58. doi: 10.1111/cas.14230

97. Paulus P, Stanley ER, Schafer R, Abraham D, Aharinejad S. ColonyStimulating Factor-1 Antibody Reverses Chemoresistance in Human MCF-7 Breast Cancer Xenografts. Cancer Res (2006) 66:4349-56. doi: 10.1158/0008-5472.CAN-05-3523

98. Zhou L, Jiang Y, Liu X, Li L, Yang X, Dong C, et al. Promotion of TumorAssociated Macrophages Infiltration by Elevated Neddylation Pathway Via NF-kappaB-CCL2 Signaling in Lung Cancer. Oncogene (2019) 38:5792-804. doi: 10.1038/s41388-019-0840-4

99. Sandhu SK, Papadopoulos K, Fong PC, Patnaik A, Messiou C, Olmos D, et al. A First-in-Human, First-in-Class, Phase I Study of Carlumab (CNTO 888), a Human Monoclonal Antibody Against CC-Chemokine Ligand 2 in Patients With Solid Tumors. Cancer Chemother Pharmacol (2013) 71:104150. doi: 10.1007/s00280-013-2099-8

100. Pienta KJ, Machiels JP, Schrijvers D, Alekseev B, Shkolnik M, Crabb SJ, et al. Phase 2 Study of Carlumab (CNTO 888), a Human Monoclonal Antibody Against CCChemokine Ligand 2 (CCL2), in Metastatic Castration-Resistant Prostate Cancer. Invest New Drugs (2013) 31:760-8. doi: 10.1007/s10637-012-9869-8

101. Nywening TM, Wang-Gillam A, Sanford DE, Belt BA, Panni RZ, Cusworth $\mathrm{BM}$, et al. Targeting Tumour-Associated Macrophages With CCR2 Inhibition in Combination With FOLFIRINOX in Patients With Borderline Resectable and Locally Advanced Pancreatic Cancer: A SingleCentre, Open-Label, Dose-Finding, Non-Randomised, Phase 1b Trial. Lancet Oncol (2016) 17:651-62. doi: 10.1016/S1470-2045(16)00078-4

102. Hughes R, Qian BZ, Rowan C, Muthana M, Keklikoglou I, Olson OC, et al. Perivascular M2 Macrophages Stimulate Tumor Relapse After Chemotherapy. Cancer Res (2015) 75:3479-91. doi: 10.1158/0008-5472.CAN-14-3587

103. Li X, Bu W, Meng L, Liu X, Wang S, Jiang L, et al. CXCL12/CXCR4 Pathway Orchestrates CSC-Like Properties by CAF Recruited Tumor Associated Macrophage in OSCC. Exp Cell Res (2019) 378:131-8. doi: 10.1016/ j.yexcr.2019.03.013

104. Bockorny B, Semenisty V, Macarulla T, Borazanci E, Wolpin BM, Stemmer $\mathrm{SM}$, et al. Bl-8040, a CXCR4 Antagonist, in Combination With Pembrolizumab and Chemotherapy for Pancreatic Cancer: The COMBAT Trial. Nat Med (2020) 26:878-85. doi: 10.1038/s41591-020-0880-x

105. Klein S, Abraham M, Bulvik B, Dery E, Weiss ID, Barashi N, et al. Cxcr4 Promotes Neuroblastoma Growth and Therapeutic Resistance Through Mir15a/16-1-Mediated ERK and BCL2/Cyclin D1 Pathways. Cancer Res (2018) 78:1471-83. doi: 10.1158/0008-5472.CAN-17-0454

106. Hassan S, Buchanan M, Jahan K, Aguilar-Mahecha A, Gaboury L, Muller WJ, et al. CXCR4 Peptide Antagonist Inhibits Primary Breast Tumor Growth, Metastasis and Enhances the Efficacy of anti-VEGF Treatment or Docetaxel in a Transgenic Mouse Model. Int J Cancer (2011) 129:225-32. doi: 10.1002/ijc.25665

107. Ishida Y, Kuninaka Y, Yamamoto Y, Nosaka M, Kimura A, Furukawa F, et al. Pivotal Involvement of the CX3CL1-CX3CR1 Axis for the Recruitment of M2 Tumor-Associated Macrophages in Skin Carcinogenesis. J Invest Dermatol (2020) 140:1951-61. doi: 10.1016/j.jid.2020.02.023

108. Koh YW, Park C, Yoon DH, Suh C, Huh J. Csf-1R Expression in TumorAssociated Macrophages is Associated With Worse Prognosis in Classical Hodgkin Lymphoma. Am J Clin Pathol (2014) 141:573-83. doi: 10.1309/ AJCPR92TDDFARISU

109. Cannarile MA, Weisser M, Jacob W, Jegg AM, Ries CH, Ruttinger D. Colony-Stimulating Factor 1 Receptor (CSF1R) Inhibitors in Cancer Therapy. J Immunother Cancer (2017) 5:53. doi: 10.1186/s40425-017-0257-y

110. Wang N, Wang S, Wang X, Zheng Y, Yang B, Zhang J, et al. Research Trends in Pharmacological Modulation of Tumor-Associated Macrophages. Clin Transl Med (2021) 11:e288. doi: 10.1002/ctm2.288

111. Yan D, Kowal J, Akkari L, Schuhmacher AJ, Huse JT, West BL, et al. Inhibition of Colony Stimulating Factor-1 Receptor Abrogates Microenvironment- 
Mediated Therapeutic Resistance in Gliomas. Oncogene (2017) 36:6049-58. doi: 10.1038/onc.2017.261

112. Shiao SL, Ruffell B, DeNardo DG, Faddegon BA, Park CC, Coussens LM. Th2-Polarized Cd4(+) T Cells and Macrophages Limit Efficacy of Radiotherapy. Cancer Immunol Res (2015) 3:518-25. doi: 10.1158/23266066.CIR-14-0232

113. Tap WD, Wainberg ZA, Anthony SP, Ibrahim PN, Zhang C, Healey JH, et al. Structure-Guided Blockade of CSF1R Kinase in Tenosynovial Giant-Cell Tumor. N Engl J Med (2015) 373:428-37. doi: 10.1056/NEJMoa1411366

114. Tap WD, Gelderblom H, Palmerini E, Desai J, Bauer S, Blay JY, et al. Pexidartinib Versus Placebo for Advanced Tenosynovial Giant Cell Tumour (ENLIVEN): A Randomised Phase 3 Trial. Lancet (2019) 394:478-87. doi: 10.1016/S0140-6736(19)30764-0

115. Butowski N, Colman H, De Groot JF, Omuro AM, Nayak L, Wen PY, et al. Orally Administered Colony Stimulating Factor 1 Receptor Inhibitor PLX3397 in Recurrent Glioblastoma: An Ivy Foundation Early Phase Clinical Trials Consortium Phase II Study. Neuro Oncol (2016) 18:557-64. doi: 10.1093/neuonc/nov245

116. von Tresckow B, Morschhauser F, Ribrag V, Topp MS, Chien C, Seetharam S, et al. An Open-Label, Multicenter, Phase I/Ii Study of JNJ-40346527, a CSF-1R Inhibitor, in Patients With Relapsed or Refractory Hodgkin Lymphoma. Clin Cancer Res (2015) 21:1843-50. doi: 10.1158/1078-0432. CCR-14-1845

117. Kumar V, Donthireddy L, Marvel D, Condamine T, Wang F, Lavilla-Alonso S, et al. Cancer-Associated Fibroblasts Neutralize the Anti-tumor Effect of CSF1 Receptor Blockade by Inducing Pmn-Mdsc Infiltration of Tumors. Cancer Cell (2017) 32:654-68. doi: 10.1016/j.ccell.2017.10.005

118. Moreau MF, Guillet C, Massin P, Chevalier S, Gascan H, Basle MF, et al. Comparative Effects of Five Bisphosphonates on Apoptosis of Macrophage Cells In Vitro. Biochem Pharmacol (2007) 73:718-23. doi: 10.1016/j.bcp. 2006.09.031

119. Germano G, Frapolli R, Belgiovine C, Anselmo A, Pesce S, Liguori M, et al. Role of Macrophage Targeting in the Antitumor Activity of Trabectedin. Cancer Cell (2013) 23:249-62. doi: 10.1016/j.ccr.2013.01.008

120. Van Acker HH, Anguille S, Willemen Y, Smits EL, Van Tendeloo VF. Bisphosphonates for Cancer Treatment: Mechanisms of Action and Lessons From Clinical Trials. Pharmacol Ther (2016) 158:24-40. doi: 10.1016/ j.pharmthera.2015.11.008

121. Junankar S, Shay G, Jurczyluk J, Ali N, Down J, Pocock N, et al. Real-Time Intravital Imaging Establishes Tumor-Associated Macrophages as the Extraskeletal Target of Bisphosphonate Action in Cancer. Cancer Discovery (2015) 5:35-42. doi: 10.1158/2159-8290.CD-14-0621

122. D'Incalci M, Zambelli A. Trabectedin for the Treatment of Breast Cancer. Expert Opin Investig Drugs (2016) 25:105-15. doi: 10.1517/13543784. 2016.1124086

123. Carminati L, Pinessi D, Borsotti P, Minoli L, Giavazzi R, D’Incalci M, et al. Antimetastatic and Antiangiogenic Activity of Trabectedin in Cutaneous Melanoma. Carcinogenesis (2019) 40:303-12. doi: 10.1093/carcin/bgy177

124. Barclay AN, Van den Berg TK. The Interaction Between Signal Regulatory Protein Alpha (Sirpalpha) and CD47: Structure, Function, and Therapeutic Target. Annu Rev Immunol (2014) 32:25-50. doi: 10.1146/annurevimmunol-032713-120142

125. Logtenberg M, Scheeren FA, Schumacher TN. The CD47-SIRPalpha Immune Checkpoint. Immunity (2020) 52:742-52. doi: 10.1016/j.immuni. 2020.04.011

126. Veillette A, Chen J. Sirpalpha-CD47 Immune Checkpoint Blockade in Anticancer Therapy. Trends Immunol (2018) 39:173-84. doi: 10.1016/ j.it.2017.12.005

127. Zhang M, Hutter G, Kahn SA, Azad TD, Gholamin S, Xu CY, et al. AntiCD47 Treatment Stimulates Phagocytosis of Glioblastoma by M1 and M2 Polarized Macrophages and Promotes M1 Polarized Macrophages In Vivo. PloS One (2016) 11:e153550. doi: 10.1371/journal.pone.0153550

128. Xiao Z, Chung H, Banan B, Manning PT, Ott KC, Lin S, et al. Antibody Mediated Therapy Targeting CD47 Inhibits Tumor Progression of Hepatocellular Carcinoma. Cancer Lett (2015) 360:302-9. doi: 10.1016/ j.canlet.2015.02.036

129. Gholamin S, Mitra SS, Feroze AH, Liu J, Kahn SA, Zhang M, et al. Disrupting the CD47-SIRPalpha Anti-Phagocytic Axis by a Humanized anti-CD47
Antibody is an Efficacious Treatment for Malignant Pediatric Brain Tumors. Sci Transl Med (2017) 9:eaaf2968. doi: 10.1126/scitranslmed.aaf2968

130. Feng M, Jiang W, Kim B, Zhang CC, Fu YX, Weissman IL. Phagocytosis Checkpoints as New Targets for Cancer Immunotherapy. Nat Rev Cancer (2019) 19:568-86. doi: 10.1038/s41568-019-0183-z

131. Lentz RW, Colton MD, Mitra SS, Messersmith WA. Innate Immune Checkpoint Inhibitors: The Next Breakthrough in Medical Oncology? Mol Cancer Ther (2021). doi: 10.1158/1535-7163.MCT-21-0041

132. van der Touw W, Chen HM, Pan PY, Chen SH. LILRB Receptor-Mediated Regulation of Myeloid Cell Maturation and Function. Cancer Immunol Immunother (2017) 66:1079-87. doi: 10.1007/s00262-017-2023-x

133. Chen HM, van der Touw W, Wang YS, Kang K, Mai S, Zhang J, et al. Blocking Immunoinhibitory Receptor LILRB2 Reprograms TumorAssociated Myeloid Cells and Promotes Antitumor Immunity. J Clin Invest (2018) 128:5647-62. doi: 10.1172/JCI97570

134. Sharma N, Atolagbe OT, Ge Z, Allison JP. LILRB4 Suppresses Immunity in Solid Tumors and is a Potential Target for Immunotherapy. J Exp Med (2021) 218:e20201811. doi: 10.1084/jem.20201811

135. Vonderheide RH. Cd40 Agonist Antibodies in Cancer Immunotherapy. Annu Rev Med (2020) 71:47-58. doi: 10.1146/annurev-med-062518-045435

136. Beatty GL, Chiorean EG, Fishman MP, Saboury B, Teitelbaum UR, Sun W, et al. CD40 Agonists Alter Tumor Stroma and Show Efficacy Against Pancreatic Carcinoma in Mice and Humans. Science (2011) 331:1612-6. doi: $10.1126 /$ science. 1198443

137. Baumann D, Hagele T, Mochayedi J, Drebant J, Vent C, Blobner S, et al. Proimmunogenic Impact of MEK Inhibition Synergizes With Agonist antiCD40 Immunostimulatory Antibodies in Tumor Therapy. Nat Commun (2020) 11:2176. doi: 10.1038/s41467-020-15979-2

138. Fitzgerald KA, Kagan JC. Toll-Like Receptors and the Control of Immunity. Cell (2020) 180:1044-66. doi: 10.1016/j.cell.2020.02.041

139. Urban-Wojciuk Z, Khan MM, Oyler BL, Fahraeus R, Marek-Trzonkowska N, Nita-Lazar A, et al. The Role of TLRs in Anti-Cancer Immunity and Tumor Rejection. Front Immunol (2019) 10:2388. doi: 10.3389/ fimmu.2019.02388

140. Liu Z, Xie Y, Xiong Y, Liu S, Qiu C, Zhu Z, et al. TLR 7/8 Agonist Reverses Oxaliplatin Resistance in Colorectal Cancer Via Directing the MyeloidDerived Suppressor Cells to Tumoricidal M1-Macrophages. Cancer Lett (2020) 469:173-85. doi: 10.1016/j.canlet.2019.10.020

141. Ji N, Mukherjee N, Morales EE, Tomasini ME, Hurez V, Curiel TJ, et al. Percutaneous BCG Enhances Innate Effector Antitumor Cytotoxicity During Treatment of Bladder Cancer: A Translational Clinical Trial. Oncoimmunology (2019) 8:1614857. doi: 10.1080/2162402X.2019.1614857

142. Aznar MA, Tinari N, Rullan AJ, Sanchez-Paulete AR, Rodriguez-Ruiz ME, Melero I. Intratumoral Delivery of Immunotherapy-Act Locally, Think Globally. J Immunol (2017) 198:31-9. doi: 10.4049/jimmunol.1601145

143. Mullins SR, Vasilakos JP, Deschler K, Grigsby I, Gillis P, John J, et al. Intratumoral Immunotherapy With TLR7/8 Agonist MEDI9197 Modulates the Tumor Microenvironment Leading to Enhanced Activity When Combined With Other Immunotherapies. J Immunother Cancer (2019) 7:244. doi: 10.1186/s40425-019-0724-8

144. Mulder W, Ochando J, Joosten L, Fayad ZA, Netea MG. Therapeutic Targeting of Trained Immunity. Nat Rev Drug Discovery (2019) 18:55366. doi: 10.1038/s41573-019-0025-4

145. Nurnberg B, Beer-Hammer S. Function, Regulation and Biological Roles of PI3Kgamma Variants. Biomolecules (2019) 9:427. doi: 10.3390/biom9090427

146. Kaneda MM, Messer KS, Ralainirina N, Li H, Leem CJ, Gorjestani S, et al. PI3Kgamma Is a Molecular Switch That Controls Immune Suppression. Nature (2016) 539:437-42. doi: 10.1038/nature19834

147. Yang L, Venneti S, Nagrath D. Glutaminolysis: A Hallmark of Cancer Metabolism. Annu Rev BioMed Eng (2017) 19:163-94. doi: 10.1146/ annurev-bioeng-071516-044546

148. Menga A, Serra M, Todisco S, Riera-Domingo C, Ammarah U, Ehling M, et al. Glufosinate Constrains Synchronous and Metachronous Metastasis by Promoting Anti-Tumor Macrophages. EMBO Mol Med (2020) 12:e11210. doi: 10.15252/emmm.201911210

149. Singh AK, Bishayee A, Pandey AK. Targeting Histone Deacetylases With Natural and Synthetic Agents: An Emerging Anticancer Strategy. Nutrients (2018) 10:731. doi: 10.3390/nu10060731 
150. Guerriero JL, Sotayo A, Ponichtera HE, Castrillon JA, Pourzia AL, Schad S, et al. Class IIa HDAC Inhibition Reduces Breast Tumours and Metastases Through Anti-Tumour Macrophages. Nature (2017) 543:428-32. doi: $10.1038 /$ nature21409

151. Di Caro G, Cortese N, Castino GF, Grizzi F, Gavazzi F, Ridolfi C, et al. Dual Prognostic Significance of Tumour-Associated Macrophages in Human Pancreatic Adenocarcinoma Treated or Untreated With Chemotherapy. Gut (2016) 65:1710-20. doi: 10.1136/gutjnl-2015-309193

152. Wanderley CW, Colon DF, Luiz J, Oliveira FF, Viacava PR, Leite CA, et al. Paclitaxel Reduces Tumor Growth by Reprogramming Tumor-Associated Macrophages to an M1 Profile in a TLR4-Dependent Manner. Cancer Res (2018) 78:5891-900. doi: 10.1158/0008-5472.CAN-17-3480

153. Shah MA, Enzinger P, Ko AH, Ocean AJ, Philip PA, Thakkar PV, et al. Multicenter Phase Ii Study of Cabazitaxel in Advanced Gastroesophageal Cancer: Association of HER2 Expression and M2-Like Tumor-Associated Macrophages With Patient Outcome. Clin Cancer Res (2020) 26:4756-66. doi: 10.1158/1078-0432.CCR-19-3920

154. Cortese N, Donadon M, Rigamonti A, Marchesi F. Macrophages At the Crossroads of Anticancer Strategies. Front Biosci (Landmark Ed) (2019) 24:1271-83. doi: 10.2741/4779

155. Galluzzi L, Buque A, Kepp O, Zitvogel L, Kroemer G. Immunogenic Cell Death in Cancer and Infectious Disease. Nat Rev Immunol (2017) 17:97-111. doi: 10.1038/nri.2016.107

156. Klug F, Prakash H, Huber PE, Seibel T, Bender N, Halama N, et al. Low-Dose Irradiation Programs Macrophage Differentiation to an iNOS(+)/M1
Phenotype That Orchestrates Effective T Cell Immunotherapy. Cancer Cell (2013) 24:589-602. doi: 10.1016/j.ccr.2013.09.014

157. van der Sluis TC, Sluijter M, van Duikeren S, West BL, Melief CJ, Arens R, et al. Therapeutic Peptide Vaccine-Induced Cd8 T Cells Strongly Modulate Intratumoral Macrophages Required for Tumor Regression. Cancer Immunol Res (2015) 3:1042-51. doi: 10.1158/2326-6066.CIR-15-0052

158. Etzerodt A, Tsalkitzi K, Maniecki M, Damsky W, Delfini M, Baudoin E, et al. Specific Targeting of CD163(+) Tams Mobilizes Inflammatory Monocytes and Promotes T Cell-Mediated Tumor Regression. J Exp Med (2019) 216:2394-411. doi: 10.1084/jem.20182124

159. Kiss M, Vande WL, Saavedra P, Lebegge E, Van Damme H, Murgaski A, et al. Illbeta Promotes Immune Suppression in the Tumor Microenvironment Independent of the Inflammasome and Gasdermin D. Cancer Immunol Res (2021) 9:309-23. doi: 10.1158/2326-6066.CIR-20-0431

Conflict of Interest: The authors declare that the research was conducted in the absence of any commercial or financial relationships that could be construed as a potential conflict of interest.

Copyright (C) 2021 Tan, Wang, Zhang, Ge, Zhong, Xia and Sun. This is an open-access article distributed under the terms of the Creative Commons Attribution License (CC BY). The use, distribution or reproduction in other forums is permitted, provided the original author(s) and the copyright owner(s) are credited and that the original publication in this journal is cited, in accordance with accepted academic practice. No use, distribution or reproduction is permitted which does not comply with these terms. 


\section{GLOSSARY}

A2R, A2 adenosine receptor; APCs, antigen-presenting cells; BCG, bacilli calmette Guerin; BM, bone marrow; BMDMs, bone-marrow-derived macrophages; CSF-1, colony-stimulating factor 1; CSF1-R, CSF-1 receptor; CD206, mannose receptor, CD163, scavenger receptor; COPD, chronic obstructive pulmonary disease; CCL, chemokine (C-C motif) ligand; CCR, chemokine (C-C motif) receptor; CXCL, CXC motif chemokine ligand; CXCR: CXC chemokine receptor; CTLs, cytotoxic CD8+ T lymphocytes; COX-2, cyclooxygenase-2; CSCs, cancer stem cells; CX3CL1, C-X3-C motif chemokine ligand 1; CX3CR1, CX3C chemokine ligand 1 receptor; DAMP, damage-associated molecular patterns; DCs, dendritic cells; EMPs, erythro-myeloid progenitors; EGF, epidermal growth factor; HB-EGF, heparinbinding EGF; EGFR, EGF receptor; ERK5, extracellularly regulated protein kinase 5; EMT, epithelial-mesenchymal transition; ECM, extracellular matrix; GS, glutamine synthetase; HSCs, hematopoietic stem cells; HUVECs, human umbilical vein endothelial cells; HDAC, histone deacetylase; HLA, human leukocyte antigen; ICD, immunogenic cell death; IL, interleukin; IFN- $\gamma$, interferon- $\gamma$; IDO, indoleamine 2,3 dioxygenase; Igs, immunoglobulins; LPS, lipopolysaccharide; ITIM, immunoreceptor tyrosine-based inhibitory motifs; LILR, leukocyte immunoglobulin-like receptor; LILRB1, leukocyte immunoglobulin-like receptor B subfamily member 1; MDSCs, myeloid-derived suppressor cells; MMPs, matrix metalloproteinases; MHC, major histocompatibility complex; MHC I, major histocompatibility complex class I; mCRPC, metastatic castration-resistant prostate cancer; MEKi, mitogenactivated protein kinase inhibitors; NO, nitric oxide; NOS2, nitric oxide synthase; NK cells, Natural killer cells; NF- $\mathrm{KB}$, nuclear factor kappa B; PDGF, platelet-derived growth factor; PEG2, prostaglandin-E2; PFS, progression free survival; ROS, reactive oxygen species; STAT3, Signal transducer and activator of transcription 3; SIRP $\alpha$, signal-regulated protein alpha; TAMs, tumor-associated macrophages; TME, tumor microenvironment; TRMs, tissue-resident macrophages; TNF$\alpha$, tumor necrosis factor- $\alpha$; TLRs, Toll-like receptors; TIE2, angiopoietin 1 receptor; Tregs, regulatory $\mathrm{T}$ cells; TRAIL, TNF-related apoptosis-inducing ligand; TGCT, Tenosynovial giant cell tumor; VEGF, vascular endothelial growth factor; VEGF-A, vascular endothelial growth factor A; VCAM1, vascular cell adhesion protein 1 . 